\title{
Kinematics Measurement and Power Requirements of Fruitflies at Various Flight Speeds
}

\author{
Hao Jie Zhu * and Mao Sun \\ Ministry-of-Education Key Laboratory of Fluid Mechanics, Beihang University, Beijing 100191, China; \\ m.sun@buaa.edu.cn \\ * Correspondence: by1505144@buaa.edu.cn
}

Received: 22 June 2020; Accepted: 11 August 2020; Published: 18 August 2020

\begin{abstract}
Energy expenditure is a critical characteristic in evaluating the flight performance of flying insects. To investigate how the energy cost of small-sized insects varies with flight speed, we measured the detailed wing and body kinematics in the full speed range of fruitflies and computed the aerodynamic forces and power requirements of the flies. As flight speed increases, the body angle decreases and the stroke plane angle increases; the wingbeat frequency only changes slightly; the geometrical angle of attack in the middle upstroke increases; the stroke amplitude first decreases and then increases. The mechanical power of the fruitflies at all flight speeds is dominated by aerodynamic power (inertial power is very small), and the magnitude of aerodynamic power in upstroke increases significantly at high flight speeds due to the increase of the drag and the flapping velocity of the wing. The specific power (power required for flight divided by insect weigh) changes little when the advance ratio is below about 0.45 and afterwards increases sharply. That is, the specific power varies with flight speed according to a J-shaped curve, unlike those of aircrafts, birds and large-sized insects which vary with flight speed according to a U-shaped curve.
\end{abstract}

Keywords: forward flight; kinematics; power requirement; fruitfly; insect

\section{Introduction}

Hovering and level-forward flight are the most common flight modes of insects. To accomplish various flight tasks in different scenarios, insects adjust the generation mechanisms of aerodynamic forces by sophisticatedly controlling the flapping motion of their wings [1]. Since flight is an energetically demanding mode of locomotion [2], there is an optimization pressure for insects to select the appropriate flight speed to economize energy consumption according to specific tasks. Previous studies have shown that the power-speed curve of flying vertebrates such as birds and bats [3,4], similar to that of helicopters, is U-shaped, which means the migration distance can be reached at its maximum when flying at some intermediate speed. However, whether the U-shaped model is suitable for insects remains unclear, because the wings of insects operate at relatively low Reynolds number and the flows around are strongly unsteady during flight, which is quite different from that of vertebrates and helicopters.

To remain airborne, the mechanical power output of insects' flight muscles must match the requirement for flapping the wings [5]. In order to investigate the variation of mechanical power with flight speed increasing, several experimental measurements have been conducted during insects' freely forward flight. Ellington et al. [6] measured the oxygen consumption rates of bumblebees flying in a wind tunnel at the speeds up to $4 \mathrm{~m} / \mathrm{s}$; it was shown that the metabolic rate only changed slightly with flight speed, suggesting that the U-shaped model may be inadequate for bumblebees. Warfvinge et al. [7] reconstructed the wake of hawkmoth flying in a wind tunnel over a speed range of $1-3.8 \mathrm{~m} / \mathrm{s}$ by using the tomographic particle image velocimetry technique, and calculated the 
mechanical power as the rate of energy added into the wake by the flapping wings; the result suggested that the power curve of hawkmoths is U-shaped.

Benefit from the measurement and calculation methods used in the above two studies, total mechanical power can be obtained without measuring the wing kinematics of insects; but these methods also have some limitations, e.g., they are difficult to acquire the time courses of instantaneous power in a wingbeat cycle. One approach to improve this situation is that using the method of computational fluid dynamics (CFD) to study the power requirement of insect flight, which requires the wing and body kinematics were accurately measured at first. However, present experimental measurements were mostly focused on hovering or single-speed flight [8-19]; there only exist three studies that measured the wing and body kinematics of an individual insect in multiple-speed forward flight [20-22]. Dudley and Ellington [20] filmed and measured the forward flight of three bumblebees using a wind tunnel and each individual had 3-4 flight speeds; since only one high-speed camera was used, the pitching angle of flapping wings could just be estimated. Willmott and Ellington [21] further measured the complete kinematics of three hawkmoths using the same wind tunnel and two high-speed cameras and each individual had 5-6 flight speeds. In these two studies, the measured maximum flight speeds were 4.5 and $5 \mathrm{~m} / \mathrm{s}$ for bumblebees and hawkmoths, respectively, which may not reach the limit of flight capability of these two insects. Recently, Meng and Sun [22] measured the wing and body kinematics in the full flight speed range of three droneflies, each had 6 flight speeds, from hovering to $8.6 \mathrm{~m} / \mathrm{s}$ with the usage of three orthogonally aligned high-speed cameras and a customized low-speed wind tunnel.

Based on the measured kinematic data of bumblebees from Dudley and Ellington [20], Wu and Sun [23] studied the trend of mechanical power with flight speed. The results demonstrated that the power required at medium speeds only decreased slightly from that at hovering and became relatively large at the measured highest speed, i.e., the power curve was approximately J-shaped. However, due to the absence of the kinematics at the potential maximum flight speed, the conceivably last rapid-growth phase of the curve was lacking.

The above brief review shows that presently there are only three species of insects (bumblebee, hawkmoth and dronefly) whose kinematic data at multiple flight speeds were measured [20-22], and previous energetic researches were all based on the data that were measured over a partial speed range $[6,7,23]$. And it has been shown that, for the medium-sized bumblebee whose wing length is about $13 \mathrm{~mm}$, the power-speed curve is probably J-shaped [23]; for the large-sized hawkmoth whose wing length is about $50 \mathrm{~mm}$, the curve is more likely U-shaped [7]. But for other insects, especially for small-sized insects (e.g., fruitfly), no research based on measured kinematics has been conducted to explore how their power cost changes with flight speed. Therefore, the shape of the power-speed curve of insects is still not yet fully understood.

In the present paper, first, the wing and body kinematics of fruitflies in forward flight were quantitatively measured using three-dimensional high-speed cinematography technique. The experiments were carried out in a wind tunnel and hence the measured speeds could be distributed in the full range of possible speeds. Then, the aerodynamic forces and torques exerted on the wings were computed by numerically solving the Navier-Stokes equations, and the inertial torques were computed analytically. On the basis of the aerodynamic and inertial torques, the mechanical power required for each flight was calculated. Therefore, what the shape of the power-speed curve is for this small-sized insect, whose wing length is about $3 \mathrm{~mm}$, can be known. Selecting fruitfly is not only due to that it is a valuable model insect which has excellent ability to hover or fly forward stably and has been extensively used in flight mechanics and other biological fields, also due to that its wing kinematics in forward flight are still quite lacking. Although the body motions in freely forward flight have been tracked when studying the flight behaviors of fruitflies under visual control [24-26], there is still no measured wing kinematics of fruitfly flying at high speed; the measured largest flight speed for fruitfly (fruitfly $F_{1}$ in Meng and Sun [14]) with detailed wing motion obtained was about $1.0 \mathrm{~m} / \mathrm{s}$, which is only a medium flight speed for fruitfly. 


\section{Materials and Methods}

\subsection{Insects and Experimental Setup}

Experiments were performed on 3-5 days old fruitflies (Drosophila virilis), which were obtained from the Laboratory of Genetics of the School of Life Science of Peking University. These flies were descendants of wild-caught females and were reared under a $12 \mathrm{~h}$ light: $12 \mathrm{~h}$ dark cycle. Each fly was starved at least $5 \mathrm{~h}$ before being used in experiments, and the laboratory temperature was controlled at about $25^{\circ} \mathrm{C}$.

Measurement of freely forward flight of insects is much difficult than hovering, especially for large-speed situation. In measuring hovering flight of fruitflies, an enclosed cubic chamber was always used $[13,27]$. While in measuring forward flight, larger chamber size is needed but also restricted by, on one hand, the operating space of experimental platform, and on the other hand, the measurement accuracy. If chamber size is not large enough, the flight of insect in it would be full of acceleration and deceleration, and the flight speed was also greatly restricted. However, increasing the length of flight chamber, which is necessary to provide sufficient space for the insect to fly forward stably, would in turn pose difficulties for setting up an experimental platform and reduce the success rate of filming. In the study of Meng and Sun [14], a large-aspect-ratio chamber and light brightness control was used to induce fruitflies to fly forward. Even so, the average flight speed of the six fruitflies they measured was only about $0.7 \mathrm{~m} / \mathrm{s}$, far less than the flight speed limit (about $2.0 \mathrm{~m} / \mathrm{s}$ ) of fruitflies [28]. Another problem for this method of measuring insects' autonomous flight in still air is that if the flight speed is relatively large, the length of the measured arena in the flying direction is much longer than those in the other two directions, resulting in many problems for high-speed camera filming, such as the reduction of imaging quality and insufficiency of depth-of-field. Thus, it was concluded that a wind tunnel must be used in measuring high-speed forward flight of insects.

In this paper, to acquire the forward flights of fruitflies, experiments were conducted with a low-speed open-jet wind tunnel, which is customized for insect flight tests. This wind tunnel has a $15 \mathrm{~cm}$ diameter circular opening and a $0.5-10 \mathrm{~m} / \mathrm{s}$ speed range. The turbulence intensity is below $1.5 \%$ and air velocities uniformity is below $2 \%$ in the test section where flights were filmed. As shown in Figure 1, the working section of the wind tunnel was enclosed by a $20 \times 20 \times 60 \mathrm{~cm}^{3}$ plexiglass flight chamber whose upstream and downstream ends were open. And a square piece of weave fabric net was used to block the downstream end to keep the insect within the flight chamber. To film the forward flight of fruitflies, three orthogonally aligned synchronized high-speed cameras (FASTCAM Mini UX100, Photron Inc., San Diego, CA, USA; 6250 frames per second, shutter speed $20 \mu$ s, resolution $1280 \times 800$ pixels) were used. The view of each camera was backlit by a $50 \mathrm{~W}$ integrated red-light emitting diode (LED; PT-121-R-C11-MPD, Luminus Devices Inc., Sunnyvale, CA, USA; luminous flux, $4000 \mathrm{~lm}$; wavelength, $632 \mathrm{~nm}$ ), and two lenses (a CCTV lens with $25 \mathrm{~mm}$ focal length and a convex lens with $50 \mathrm{~mm}$ focal length) were used to make the light uniform. When tested insect was observed flying steadily in the filming area (intersecting field of views of the three cameras, approximately $3.5 \times 3.5 \times 3.5 \mathrm{~cm}^{3}$ ), the synchronized cameras were manually triggered. The filming area was set at the position that $11 \mathrm{~cm}$ from the opening and on the central line of the wind tunnel.

Taking advantage of phototaxis of insects, Dudley and Ellington [20] and Meng and Sun [22] both used an ultraviolet fluorescent light to elicit bumblebees and droneflies to fly in a wind tunnel and successfully measured the forward flight kinematics. Thus, in the present paper, we also employed this approach to induce fruitflies by position a $40 \mathrm{~W}$ ultraviolet fluorescent light on the roof of the chamber and near the opening of the wind tunnel (Figure 1). Most of the light tube was masked out, leaving only a small part, about $3 \mathrm{~cm}$ in length, to be visible. Through observation, we found that fruitfly indeed flied in the wind tunnel toward the location of ultraviolet light, but the flight was always unstable and not keeping on the center line of the flight chamber, such that other means were needed to stabilize the flight. 


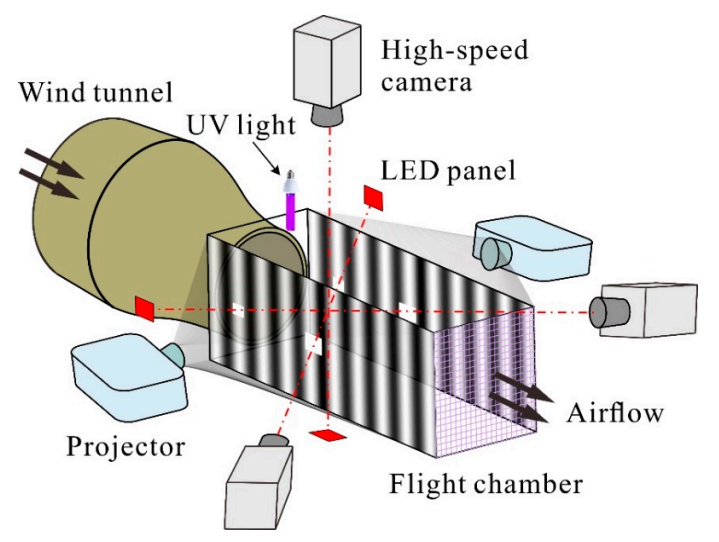

Figure 1. Sketch of filming system.

Alternatively, moving background of sine grating or random dot patterns were usually applied in the investigation of vision-based control in banked maneuvering [29] and forward flight [26]. In this paper, we applied this visual speed control method, using moving sine gratings to provide optical flow corresponding to the preferred speed of fruitflies, to control their upwind speed and stabilize them on the center line of the wind tunnel. An open-source psychophysics toolbox (Psychtoolbox 3.0) on Matlab platform (Matlab v. 9.0, The Mathworks, Inc., Natick, MA, USA) was used to accurately generate and control the visual stimuli. Then via two digital light processing (DLP) projectors (ViewSonic PJD7831HDL, ViewSonic China, Shanghai, China), the rendered sine grating stripes were displayed onto the sidewalls of the flight chamber (Figure 1). The velocity of sine grating stripes was set at $0.2 \mathrm{~m} / \mathrm{s}$, because under this visual surround, the flight speed relative to the ground of fruitfly is very small [25]. Finally, with ultraviolet light stimulating to fly upwind and moving patterns controlling the speed and stabilizing the flight, the measurement of forward flight of fruitflies can be achieved.

\subsection{Filming Procedures}

At the beginning of each experiment, the high-speed cameras were switched on and kept in the recording mode. Then the wind tunnel was turned on and waiting for about half a minute to stabilize the wind before a fruitfly was introduced into the flight chamber at the downstream end (Figure 2).

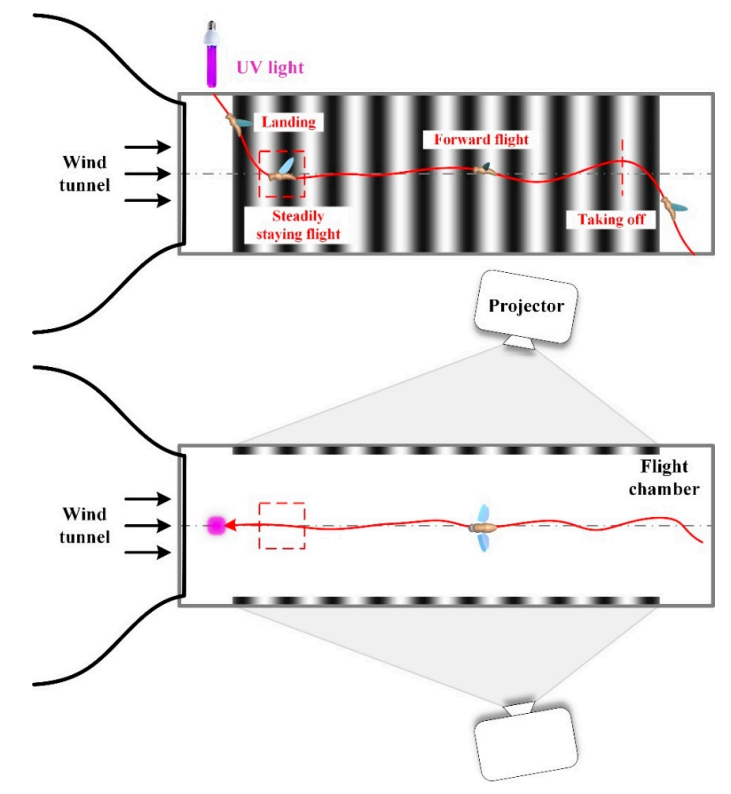

Figure 2. Diagram of the full forward-flight process of fruitflies in the flight chamber. 
The ultraviolet light was flickered on and off until the fruitfly began to take off from the rear of the flight chamber, and then the light was left on to avoid disturbing the flight. Meanwhile, the moving grating stripes were projected onto the side walls of the flight chamber. Since enticed by the ultraviolet light and oriented by the moving gratings, the tested fruitfly flew forward steadily at a low ground-speed in the working section (Figure 2).

The closer to the ultraviolet light the insect is, the lower the flight speed. When the insect reached the filming area that beneath the ultraviolet light, it stayed for several seconds (Figure 2); this is the best time to trigger the cameras so that steady forward flight can be filmed. As the flight was coming to an end, the insect adjusted its flying attitude and flew upward towards the location of the ultraviolet light to land (Figure 2). Immediately after the filming process had finished (approximately $0.89 \mathrm{~s}$ ), the ultraviolet light was turned off and the moving grating stripes were stopped. After ensuring the insect was returned to the rear of the flight chamber to rest, the filmed images were downloaded from the high-speed cameras to computer.

The key point to the achievement of the filming was the existence of the staying stage. Based upon this behavioral observation, the filming area of high-speed cameras could be preset at the staying location accordingly (Figure 2), which can greatly improve the success rate of filming. The fruitfly was almost stationary relative to the flight chamber when it was staying, thus the flight speed was approximately equal to the wind speed.

At least three flight speeds distributed in the full speed range were filmed for each fruitfly, and the experiments were begun with the measurement of the flight at the maximum speed. According to Vogel [28], the maximum flight speed of fruitflies is about $2.0 \mathrm{~m} / \mathrm{s}$, such that the wind speed was first set around this value to check whether the tested fruitflies could take off and fly forward steadily or not; if not, the wind speed was continuously lowered until it can. Thus, the filmed flight could be regarded as at the maximum speed of the tested fruitfly. Next, the wind speed was set to an intermediate speed (e.g., about $1.0 \mathrm{~m} / \mathrm{s}$ ) and the entire filming process was repeated. Last, the near-hovering flight was filmed with the visual control system and wind tunnel turned off. The total experimental time for each fruitfly was generally less than $40 \mathrm{~min}$.

\subsection{Measurement of Morphological and Kinematic Data}

The method of measuring morphological parameters was same as that given in Mou et al. [30]. Once the filming events had finished, the tested fruitfly was anaesthetized with ethyl acetate vapor. The total mass $(m)$ of the insect was measured to an accuracy of $\pm 0.01 \mathrm{mg}$. The wing planform and the body in dorsoventral and lateral views were scanned using a scanner (Epson Scanner V370; Epson (China) Co., Ltd., Beijing, China; resolution $3600 \times 3600 \mathrm{dpi}$ ) and hence the wing and body shapes can be extracted, as shown in Figure 3.

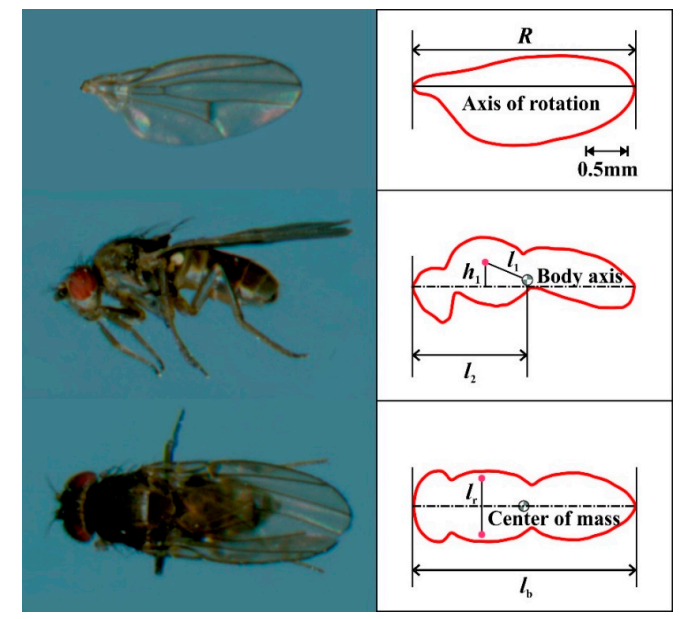

Figure 3. Morphological parameters of the wing and body of an insect. 
Then, the morphological parameters of the insect can be measured or computed based on the measured data, including wing length $(R$, the distance between wing base and wing tip), area of one wing $(S)$, mean wing chord $(c)$, radius of the second moment of wing area $\left(r_{2}\right)$, body length $\left(l_{\mathrm{b}}\right)$, distance between the wing roots $\left(l_{\mathrm{r}}\right)$, distance between the wing-root axis and the center of mass $\left(l_{1}\right.$, the center of mass is determined by assuming the body of the insect is a body of revolution with uniform density), distance between the wing-root axis and the body axis $\left(h_{1}\right)$, distance from the anterior end of the body to the center of mass $\left(l_{2}\right)$.

After all the required morphological parameters were obtained, the wing was modeled as a flat-plate with the outline extracted from the scanned cut-off wing (Figure 3), and the body was modeled as a rigid-body represented by two perpendicular lines (the line linking the head and the end of the abdomen and the line linking the two wing hinges, see Figure 4). Then the wing and body models were used in a self-developed MatLab toolbox, which is a realization of the method of three-dimensional reconstruction (see Mou et al. [30] for detailed description and error analysis), to measure the wing and body kinematics. Within the interactive graphic user interface of this toolbox, the positions and orientations of the models of the body and two wings were adjusted manually until the best overlap between their projections and the displayed frames recorded by three cameras was achieved (Figure 4); at this point, the positions and orientations of these models could be regarded as that of their real counterparts.
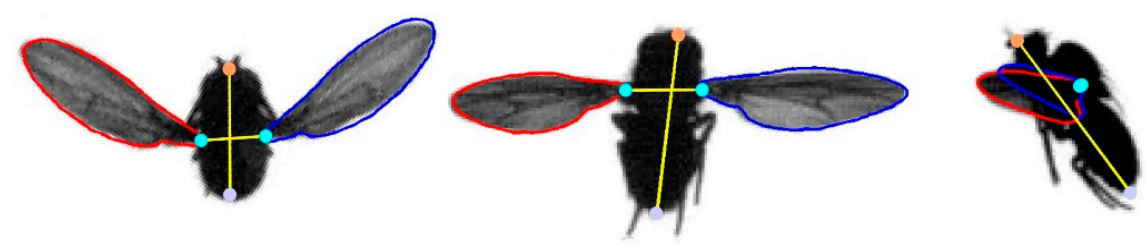

Figure 4. Example of three-dimensional reconstruction.

For a fruitfly flying at a given speed, about 35 pictures were taken during each wingbeat. After measuring frame by frame, the wing and body motions in about five wing strokes were digitized. In order to clearly describe the wing kinematics of the fruitflies, the method given by Ellington [8] was employed, and two coordinate systems and following definitions were introduced. In a reference frame that moved with the body of the fly, the discrete wing-tip trace of both of the left and right wings were projected onto the symmetric plane of the body, and a linear regression line of the projections could be determined using least square method. The plane paralleled to the above regression line and passed the wing base is defined as the stroke plane. Thereafter, two coordinate systems, oxyz and $o_{1} x_{1} y_{1} z_{1}$, are brought in. For the lab-fixed coordinate system oxyz, the origin locates at the center of the body initially, $x$-axis points forward horizontally, $y$-axis points to the right of the body horizontally and $z$-axis points downward vertically (Figure 5). For the body-fixed coordinate system $o_{1} x_{1} y_{1} z_{1}$, the origin locates at the wing base, the $x_{1}-y_{1}$ plane coincides with the stroke plane and $y_{1}$-axis coincides with $y$-axis, and $z_{1}$-axis is normal to the stroke plane (Figure 5). The inclination angle of the stroke plane about the horizontal is referred to as the stroke plane angle $(\beta)$, and the angle between the body axis and the horizontal is referred to as the body angle $(\chi)$, thus $\beta+\chi$ represents the included angle of the body axis and the stroke plane (Figure $5 a$ ).

The orientation of a flat-plate wing relative to the stroke plane can be determined by three Euler angles: the stroke angle $(\phi)$, the deviation angle $(\theta)$ and the pitch angle $(\psi)$. As shown in Figure $5 \mathrm{~b}$, $\phi$ is defined as the angle between the projection of the wing axis (the line passing wing base and wing tip) onto the stroke plane and $y_{1}$-axis; $\theta$ is defined as the angle between the wing axis and its projection onto the stroke plane; $\psi$ is defined as the angle between the local wing chord and $l$ (a line which is perpendicular to the wing axis and parallel to the stroke plane). 

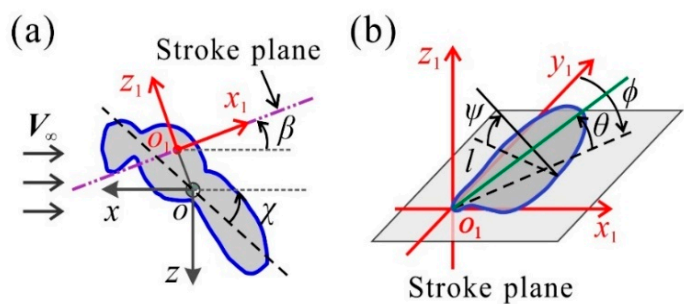

Figure 5. Coordinate systems and Euler angles of a flapping wing. (a) The lab-fixed coordinate system; (b) The body-fixed coordinate system and Euler angles.

Some other kinematic parameters of the flapping wing are as follows: $f$, the wingbeat frequency; $\Phi$, the stroke amplitude, defined as $\Phi=\phi_{\max }-\phi_{\min }\left(\phi_{\max }\right.$ and $\phi_{\min }$ are the maximum and minimum values of $\phi$, respectively); $\bar{\phi}$, the mean stroke angle, defined as $\bar{\phi}=\left(\phi_{\max }+\phi_{\min }\right) / 2 ; d / u$, the ratio of downstroke duration to upstroke duration (when $\phi$ changes from $\phi_{\max }$ to $\phi_{\min }$, the wing is referred to be in downstroke; in reverse, in upstroke). For forward flight, the advance ratio is defined as the ratio of the flight speed to the mean flapping velocity at wing tip, i.e., $J=V_{\infty} / 2 \Phi f R$, where $V_{\infty}$ is the flight speed.

\subsection{Evaluation of the Aerodynamic Forces and Mechanical Power}

The unsteady flow around the flapping wings was computed by numerically solving the incompressible Navier-Stokes equations. Previous studies have shown that for fruitfly, the aerodynamic interaction effect between the wings and the body was very small: the changes of aerodynamic forces acting on the wings with or without the presence of the body was less than $2 \%$ at hovering $[31,32]$ and $4.5 \%$ at forward flight ( $J$ was up to 0.67 with idealized wing motions) [33]. Therefore, only two wings were considered in the present CFD model, and the wing was modeled as a flat-plate with rounded leading and trailing edges. The thickness of the wing model is set at $3 \%$ of the wing chord length.

Selecting $c, U$ (the mean flapping velocity at $r_{2}$ for near hovering flight, defined as $U=2 \Phi_{0} f_{0} r_{2}$, where the subscript ' 0 ' denotes the near hovering flight) and $c / U$ as reference length, velocity and time, respectively, the dimensionless Navier-Stokes equations are as follows:

$$
\begin{gathered}
\nabla \cdot \mathbf{u}=0 \\
\frac{\partial \mathbf{u}}{\partial t^{*}}+\mathbf{u} \cdot \nabla \mathbf{u}=-\nabla p+\left(\frac{1}{R e}\right) \nabla^{2} \mathbf{u}
\end{gathered}
$$

where $\mathbf{u}$ is the non-dimensional fluid velocity field, $t^{*}$ is the non-dimensional time, $p$ is the non-dimensional fluid pressure, $R e$ is the Reynolds number, $\nabla$ is the gradient operator and $\nabla^{2}$ is the Laplacian operator.

The flow solver is the same as that in some previous studies of our group [14,16], which is based on the method of artificial compressibility developed by Rogers et al. [34,35]. In solving the equations, the method of moving overset grids is used. As shown in Figure 6, there is a body-fitted curvilinear grid for each wing which extends a relatively short distance (2.5c in the normal direction), and a background Cartesian grid which extends to the far field boundary of the domain $(20 \mathrm{c}$ in all of the $x, y, z$ directions at near-hovering, and extends to $30 \mathrm{c}$ along the downstream (negative $x$ ) direction at the maximum-speed flight). The wing grids capture such as boundary layers, separated vortices and vortex/wing interactions, and the background grid carries the solution to the far field. The two grids exchange flow information through their overlapped regions. The wing grid is $\mathrm{O}-\mathrm{H}$ type and generated using a Poisson solver which is based on the algorithm of Hilgenstock [36]. The background Cartesian grid is generated algebraically. 


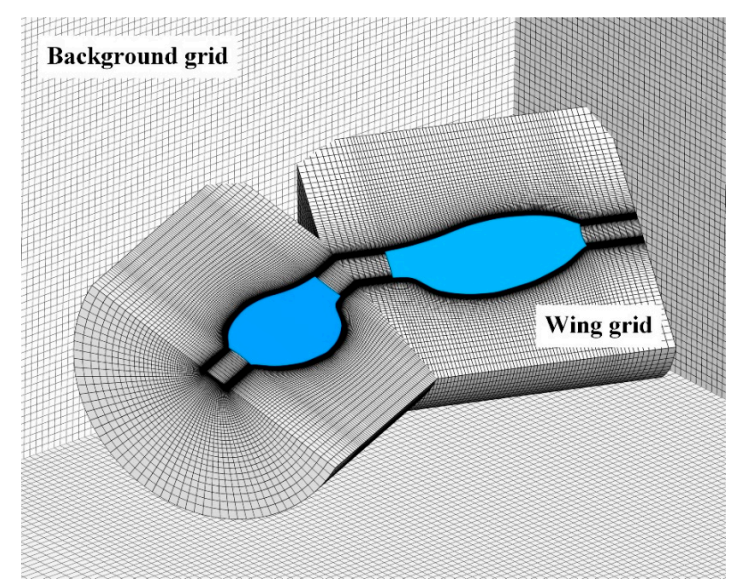

Figure 6. Portions of computational grids.

Once the Navier-Stokes equations were solved, the aerodynamic forces and moments acting on the wing were calculated by integrating the pressure and the viscous stress over the wing surface at a time step. Vertical force $(V)$ and thrust $(T)$ of a wing are defined as the components of the total aerodynamic force along the negative $z$ and positive $x$ direction, respectively; lift $(L)$ is defined as the component perpendicular to the stroke plane and along the $z_{1}$ direction; drag $(D)$ is defined as the component in the stroke plane and along the direction that opposite to the flapping movement $(\dot{\phi})$. The corresponding force coefficients, denoted by $C_{\mathrm{V}}, C_{\mathrm{T}}, C_{\mathrm{L}}$ and $C_{\mathrm{D}}$ are defined as: $C_{\mathrm{V}}=V / 0.5 \rho U^{2} S$, etc., where $\rho$ is the fluid density. The relationship between these forces is shown in Figure 7 and their transformation formulas are as follows:

$$
\begin{gathered}
C_{\mathrm{L}}=C_{\mathrm{V}} \cdot \cos \beta+C_{\mathrm{T}} \cdot \sin \beta \\
C_{\mathrm{D}}=\left(C_{\mathrm{V}} \cdot \sin \beta-C_{\mathrm{T}} \cdot \cos \beta\right) \cdot \cos \phi
\end{gathered}
$$

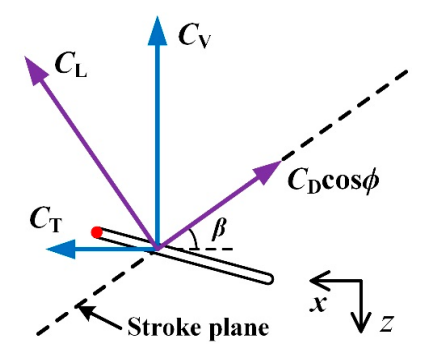

Figure 7. Relationship between aerodynamic force coefficients.

The aerodynamic and inertial moments around the wing-root are denoted as $\boldsymbol{M}_{\mathrm{a}}$ and $\boldsymbol{M}_{\mathrm{i}}$, respectively. The mechanical power $(P)$ of a flapping wing is produced by the flight muscles to overcome $\boldsymbol{M}_{\mathrm{a}}$ and $\boldsymbol{M}_{\mathrm{i}}$, and it can be calculated by:

$$
\begin{aligned}
P & =\left(\boldsymbol{M}_{\mathrm{a}}+\boldsymbol{M}_{\mathrm{i}}\right) \cdot \boldsymbol{\Omega} \\
& =\boldsymbol{M}_{\mathrm{a}} \cdot \boldsymbol{\Omega}+\boldsymbol{M}_{\mathrm{i}} \cdot \boldsymbol{\Omega} \\
& =P_{\mathrm{a}}+P_{\mathrm{i}}
\end{aligned}
$$

where $\Omega$ is the angular velocity vector of the wing, $P_{\mathrm{a}}=\boldsymbol{M}_{\mathrm{a}} \cdot \Omega$ and $P_{\mathrm{i}}=\boldsymbol{M}_{\mathrm{i}} \cdot \Omega$ are the aerodynamic and inertial power of the wing, respectively.

As $M_{\mathrm{a}}$ has already been computed using the force distribution obtained from the flow computation and $\Omega$ is also known from the measured kinematics, the aerodynamic power $P_{\mathrm{a}}$ can be readily calculated. However, the inertial power $P_{\mathrm{i}}$ still cannot be straightforwardly calculated, because the mass $\left(m_{\mathrm{w}}\right)$ and 
the radius of gyration $\left(r_{2, \mathrm{~m}}\right)$ of the wing required to determine $\boldsymbol{M}_{\mathrm{i}}$ are unknown for the tested fruitflies. To deal with this problem, the wing-to-body mass ratio $\left(m_{\mathrm{w}} / m=0.24 \%\right)$ and the non-dimensional radius of gyration $\left(r_{2, \mathrm{~m}} / R=0.48\right)$ that had been measured in Vogel [28] for the same species, Drosophila virilis, are used in the present paper. Because the venation is typically denser near the leading edge, the wing-mass mostly distributed near the axis of flip-rotation which is close to the leading edge; thus, it is reasonable to neglect the moment of inertia of the wing-mass about the axis of flip rotation. The moments of inertial about the other two axes (denoted as $I_{\mathrm{W}}$ ) are approximately the same and can be computed by $I_{\mathrm{W}}=\mathrm{emphm}_{\mathrm{w}} r_{2, \mathrm{~m}}{ }^{2}$. With $I_{\mathrm{W}}$ obtained and wing acceleration computed from $\Omega, M_{\mathrm{i}}$ thus $P_{\mathrm{i}}$ can be calculated. The coefficients of the aerodynamic and inertial power of a flapping wing are defined as $C_{\mathrm{Pa}}=P_{\mathrm{a}} / 0.5 \rho U^{3} S$ and $C_{\mathrm{Pi}}=P_{\mathrm{j}} / 0.5 \rho U^{3} S$, respectively.

\section{Results and Discussion}

\subsection{Measured Morphological Parameters and Wing and Body Motions}

In the filming experiments, the flight sequences of 24 fruitflies with at least three flight speeds were recorded. After carefully scrutinizing all the flight films of these fruitflies, we found that some fruitflies were unable to maintain steady flight under large wind-speed condition and hence their flights were accompanied with rolling or yawing motion; and some fruitflies were performing climbing flight towards the location of the ultraviolet light, not performing desired level-forward flight, when the wind speed was not very large. Therefore, for most of these fruitflies, at least one forward flight was inadequately steady or was not performing level-forward flight. Eventually, four individuals which had three steady flights were selected for further kinematic measurement. They are denoted as FF1, FF2, FF3 and FF4, respectively, and their morphological parameters are listed in Table 1.

Table 1. Morphological parameters of the fruitflies.

\begin{tabular}{cccccccccc}
\hline Individual & $\boldsymbol{m}(\mathbf{m g})$ & $c(\mathbf{m m})$ & $\boldsymbol{R}(\mathbf{m m})$ & $\boldsymbol{r}_{\mathbf{2}} / \boldsymbol{R}$ & $\boldsymbol{l}_{\mathbf{b}}(\mathbf{m m})$ & $\boldsymbol{l}_{\mathbf{r}} / l_{\mathbf{b}}$ & $\boldsymbol{l}_{\mathbf{1}} / l_{\mathbf{b}}$ & $\boldsymbol{h}_{\mathbf{1}} / l_{\mathbf{b}}$ & $\boldsymbol{l}_{\mathbf{2}} / \boldsymbol{l}_{\mathrm{b}}$ \\
\hline FF1 & 1.75 & 0.93 & 3.31 & 0.59 & 3.31 & 0.24 & 0.17 & 0.12 & 0.47 \\
FF2 & 1.22 & 0.89 & 3.05 & 0.59 & 3.33 & 0.28 & 0.15 & 0.10 & 0.45 \\
FF3 & 1.79 & 1.04 & 3.35 & 0.58 & 3.60 & 0.25 & 0.18 & 0.14 & 0.47 \\
FF4 & 2.07 & 0.98 & 3.30 & 0.58 & 3.68 & 0.27 & 0.21 & 0.15 & 0.47 \\
\hline
\end{tabular}

It is noteworthy that the above steady and balanced flights are the controlled results of insects' sensory-motor system. Previous studies [37-40] have shown that the hovering and forward flights of insects are inherently unstable, and as flight speed increases, the longitudinal instability of insect flight becomes stronger and stronger, but the inherently unstable flights of insects are controllable [37,41], counting on the fast response of sensory-motor system. That is the reason why we could capture steady forward flights of fruitflies in our experiments.

The image sequences at several instants in a flapping cycle filmed by one high-speed camera for FF1 flying at three speeds are demonstrated in Figure 8 (the corresponding video sequences are provided in the Supplementary Materials). It can be seen that the flying attitude changes with flight speed obviously: at $V_{\infty}=0.16 \mathrm{~m} / \mathrm{s}$ (near hovering), the body tilted at a relatively large angle and the wing tip position when the wing flaps to its farthest behind the body is approximately at the same altitude with that ahead the body; while as the flight speed increases, the inclined angle of the body becomes smaller and the farthest position of the wing tip behind the body becomes much higher than that ahead the body. 


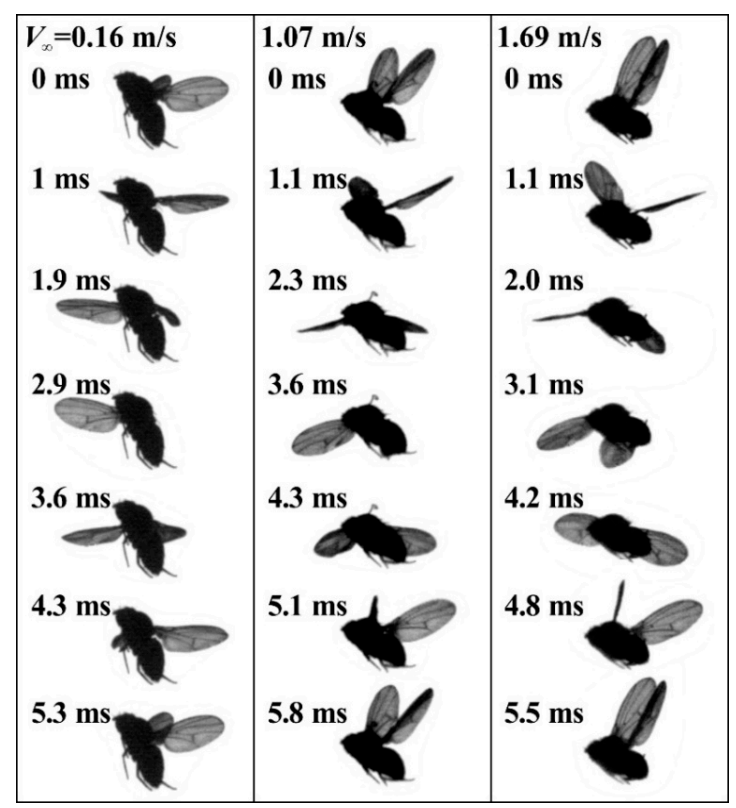

Figure 8. The filmed image sequences of FF1 flying at three flight speeds.

These observations are consistent with the measured data of the body angle $(\chi)$ and the stroke plane angle $(\beta)$ for all the four fruitflies plotted in Figure 9. From the scatters plot in Figure 9a, it can be clearly observed that $\chi$ decreases and $\beta$ increases with flight speed increasing in the speed range of the present study $\left(V_{\infty} \approx 0-2 \mathrm{~m} / \mathrm{s}\right)$, and the reduction rate of $\chi$ and the and growth rate of $\beta$ are significantly decreased when the flight speed exceeds about $1.3 \mathrm{~m} / \mathrm{s}$. While as seen from Figure $9 \mathrm{~b}$, another obvious feature is that the angle between the stroke plane and body axis, $\beta+\chi$, only fluctuates slightly, indicating that the position of the stroke plane relative to the body almost remains unchanged in the full speed range for fruitflies. These results are similar to that of bumblebees [20], hawkmoths [21] and droneflies [22] in their own measured speed ranges.
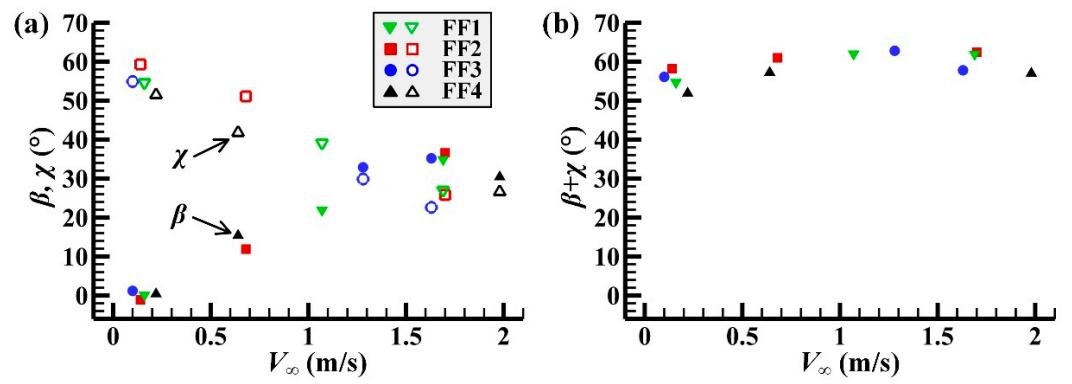

Figure 9. The variations of (a) $\beta$ and $\chi$ and (b) $\beta+\chi$ of FF1-FF4 at various flight speeds.

The variation of the stroke plane angle as functions of flight speed is highly correlated to the wing movements at each flight speed. In order to examine the wing motions, we take the flight of FF1 flying at $V_{\infty}=1.07 \mathrm{~m} / \mathrm{s}$ as an example. The measured time histories of the three Euler angles, $\phi, \theta$ and $\psi$, of the left and right wings during a period of about $26 \mathrm{~ms}$ are plotted in Figure 10a. To eliminate the high-order noise and obtain the smooth data that can be used for numerical computation, the original data of $\phi, \theta$ and $\psi$ are first averaged between left and right wings, and then are phase-averaged over five wingbeat cycles; finally, the phase-averaged data are filtered with a fifth-order low-pass Butterworth filter (the cut-off frequency are 400, 800 and $1600 \mathrm{~Hz}$ for $\phi, \theta$ and $\psi$, respectively). For convenience of description, we define a non-dimensional time, $\tau$, in a wingbeat cycle that $\tau=0$ is the start time of a downstroke and $\tau=1$ is the end time of the subsequent upstroke. The filtered Euler angles with error bands (mean \pm s.d.) as functions of $\tau$ are given in Figure 10b. 


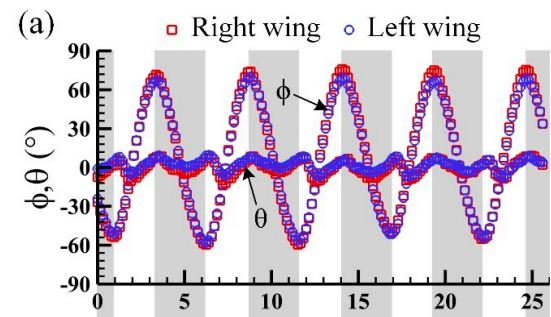

(b)
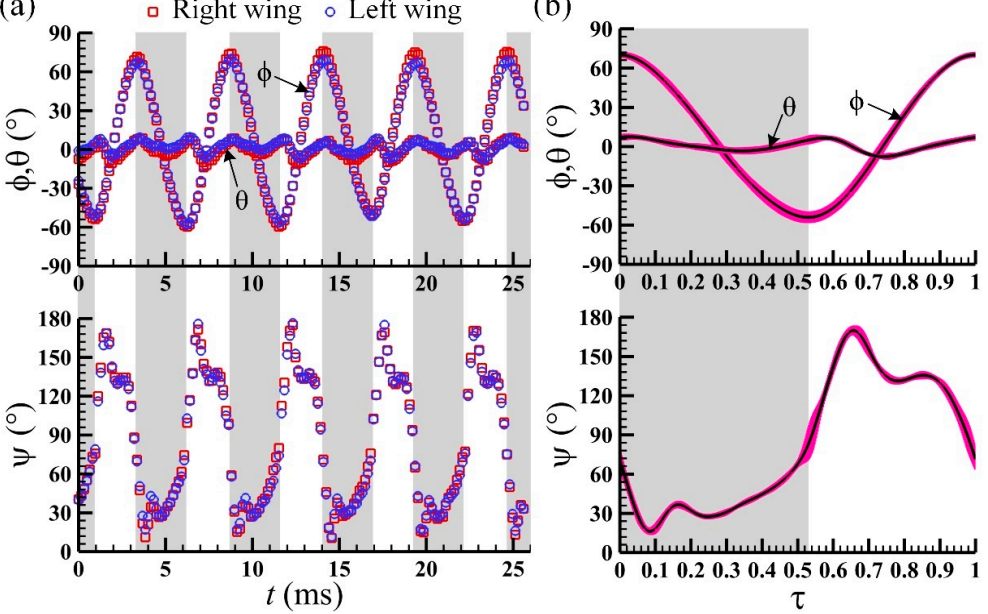

Figure 10. The measured wing kinematics of FF1 in forward flight at $V_{\infty}=1.07 \mathrm{~m} / \mathrm{s}$. (a) The original data of instantaneous Euler angles. $\phi$, the stroke angle; $\theta$, the deviation angle; $\psi$, the pitch angle. (b) The filtered values of $\phi, \theta$ and $\psi$ as function of non-dimensional period (mean \pm s.d.; $\mathrm{n}=5$ wingbeats).

The wing kinematic results of FF1 at all three flight speeds are given in Figure 11 (the corresponding results of FF2-FF4 are given in Figures A1-A3 in Appendix A, respectively). It can be seen that the magnitude of stroke angle is much larger than deviation angle (Figure 11a), such that the wing-tip paths are generally flat open loops (Figure 11c). In most of the wingbeat cycle, the trajectory of upstroke is below that of downstroke at each flight speed, except at the beginning of upstroke that one-time crossover occurs due to the wing first elevates a little and then descends ( $\theta$ increases slightly and then decreases in Figure 11a). The corresponding results of other three insects are similar.
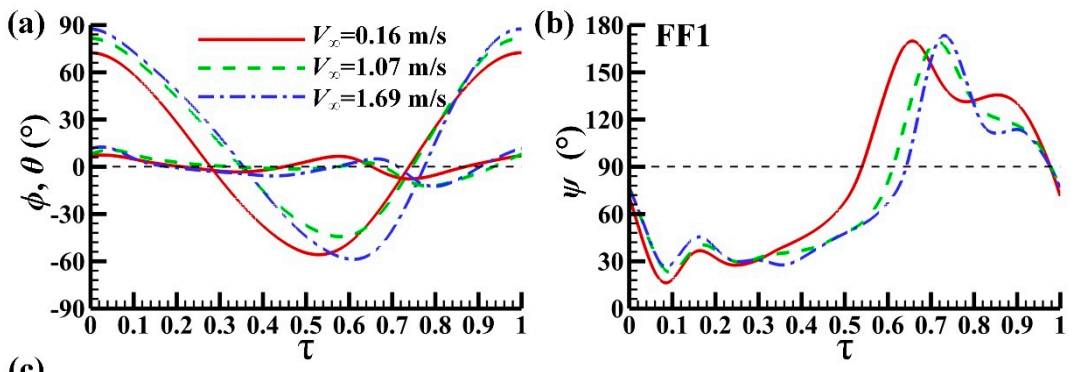

(c)

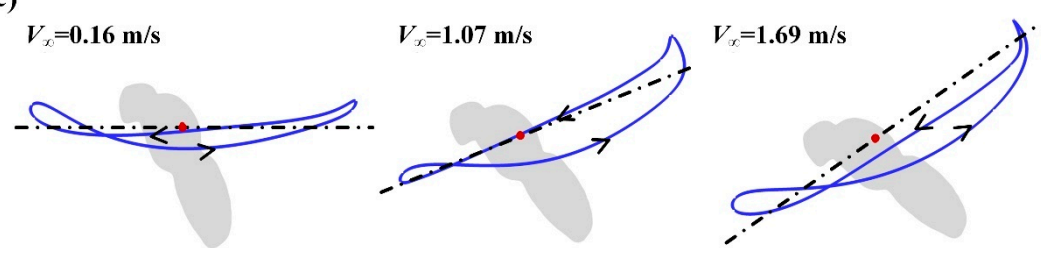

Figure 11. Time courses of (a) $\phi$ and $\theta$ and (b) $\psi$ for FF1 in one wingbeat cycle at three flight speeds. (c) The wing-tip paths relative to the stroke plane determined by $\phi$ and $\theta$ at each flight speed (the stroke plane is indicated by the dash-dot line and the wing-root is indicated by a red dot).

As shown in Figure 11b, the wing pitches up (supination) in the end of downstroke and in the beginning of the subsequent upstroke and pitches down (pronation) in the end of upstroke and in the beginning of the subsequent downstroke. To clearly describe the variation of the pitch angle in one cycle, the geometrical angle of attack $(\alpha)$ of the wing-section is defined: $\alpha=\psi$ in downstroke and $\alpha=180^{\circ}-\psi$ in upstroke. Figure 12 gives the time courses of $\alpha$ in a wingbeat cycle for FF1 at all three flight speeds (the same sets of data for the other three fruitflies are presented in Figure A4 in Appendix A). 


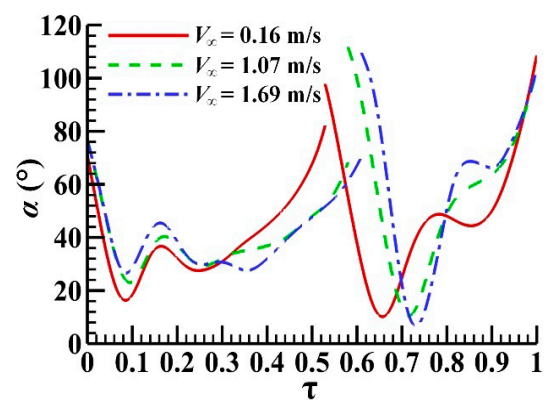

Figure 12. Time courses of $\alpha$ in a wingbeat cycle for FF1 at three flight speeds.

From Figure 12, we can see that, when near hovering $\left(V_{\infty}=0.16 \mathrm{~m} / \mathrm{s}\right)$, the wing first fast pitches down to a small $\alpha$ (from about $100^{\circ}$ to about $10^{\circ}$ ) at the beginning of upstroke ( $\tau \approx 0.53-0.66$ ); then it rapidly pitches up to a large $\alpha$ (about $\left.45^{\circ}\right)$ in a relatively short time $(\tau \approx 0.66-0.75)$ and maintains this angle in the mid-portion of upstroke $(\tau \approx 0.75-0.9)$; near the end of upstroke $(\tau \approx 0.9-1)$, the wing continues to pitch up to that $\alpha$ reaches about $110^{\circ}$. Similarly, the pitch-down and the pitch-up motions also exist in downstroke but the magnitudes that $\alpha$ changes are smaller (Figure 12). In addition, at medium $\left(V_{\infty}=1.07 \mathrm{~m} / \mathrm{s}\right)$ and high $\left(V_{\infty}=1.69 \mathrm{~m} / \mathrm{s}\right)$ flight speeds, the variation of $\alpha$ in a wingbeat cycle are similar to that at near-hovering flight (Figure 12). It can be observed that the value of $\alpha$ in the middle upstroke is generally larger than that in the middle downstroke at all flight speeds of FF1, and $\alpha$ in the middle upstroke increases with flight speed greatly (about $45^{\circ}, 60^{\circ}$ and $68^{\circ}$ at $V_{\infty}=0.16,1.07$ and $1.69 \mathrm{~m} / \mathrm{s}$, respectively) while that in the middle downstroke almost remains unchanged (about $30^{\circ}$ ). The above results are also true for the other three insects.

According to the definitions in Section 2.3, some periodical parameters of the wing motions computed from the kinematic data (Figures 11 and A1, Figures A2 and A3 in Appendix A) are listed in Table 2, including the wingbeat frequency $(f)$, the stroke amplitude $(\Phi)$, the mean stroke angle $(\bar{\phi})$, the ratio of downstroke duration to upstroke duration $(d / u)$ and the advance ratio $(J)$. For convenience, the stroke plane angle $(\beta)$ and the body angle $(\chi)$ are also listed in Table 2.

Table 2. Kinematic parameters of the fruitflies.

\begin{tabular}{|c|c|c|c|c|c|c|c|c|}
\hline Individual & $V_{\infty}(\mathrm{m} / \mathrm{s})$ & $f(\mathrm{~Hz})$ & $\Phi\left(^{\circ}\right)$ & $\phi\left(^{\circ}\right)$ & $\beta\left({ }^{\circ}\right)$ & $x\left(^{\circ}\right)$ & $d / u$ & $J$ \\
\hline \multirow{3}{*}{ FF1 } & 0.16 & $187.9 \pm 0.2$ & $126.3 \pm 2.4$ & $8.2 \pm 1.1$ & 0.1 & 54.6 & 1.12 & 0.06 \\
\hline & 1.07 & $171.4 \pm 0.1$ & $126.8 \pm 0.9$ & $18.0 \pm 0.8$ & 21.9 & 39.1 & 1.38 & 0.45 \\
\hline & 1.69 & $182.0 \pm 0.1$ & $147.1 \pm 1.1$ & $15.2 \pm 0.8$ & 34.9 & 27.0 & 1.57 & 0.53 \\
\hline \multirow{3}{*}{ FF2 } & 014 & $161.2 \pm 0.1$ & $146.0 \pm 2.2$ & $5.1 \pm 0.7$ & -1.1 & 59.3 & 1.19 & 0.06 \\
\hline & 0.68 & $156.3 \pm 0.2$ & $140.9 \pm 1.4$ & $14.5 \pm 1.2$ & 11.9 & 51.1 & 1.33 & 0.27 \\
\hline & 1.70 & $156.9 \pm 0.1$ & $161.9 \pm 1.6$ & $11.3 \pm 0.6$ & 36.6 & 25.8 & 1.59 & 0.65 \\
\hline \multirow{3}{*}{ FF3 } & 0.10 & $184.0 \pm 0.2$ & $122.2 \pm 1.6$ & $6.7 \pm 1.1$ & 1.2 & 54.9 & 1.08 & 0.04 \\
\hline & 1.28 & $189.1 \pm 0.1$ & $134.3 \pm 1.0$ & $12.1 \pm 0.5$ & 32.9 & 29.9 & 1.27 & 0.44 \\
\hline & 1.63 & $181.3 \pm 0.1$ & $144.2 \pm 1.2$ & $13.4 \pm 0.8$ & 35.2 & 22.6 & 1.34 & 0.54 \\
\hline \multirow{3}{*}{ FF4 } & 0.22 & $172.5 \pm 0.3$ & $142.8 \pm 2.8$ & $10.8 \pm 1.4$ & 0.4 & 51.5 & 1.14 & 0.08 \\
\hline & 0.64 & $170.7 \pm 0.1$ & $133.5 \pm 1.3$ & $14.8 \pm 1.3$ & 15.4 & 41.8 & 1.18 & 0.25 \\
\hline & 1.98 & $174.2 \pm 0.2$ & $164.0 \pm 0.9$ & $14.4 \pm 0.9$ & 30.4 & 26.6 & 1.44 & 0.62 \\
\hline
\end{tabular}

It can be seen that $f$ only changes slightly, within $9 \%$, with flight speed increasing, which is same as that of bumblebees [20] and hawkmoths [21]. Based on the results of the stroke amplitude listed in Table 2, increments of the stroke amplitude $(\Delta \Phi)$ can be computed by subtracting the $\Phi$ at near-hovering from that at other flight speeds for each fruitfly. Plotted in Figure 13 are $\Delta \Phi$ and $\bar{\phi}$ of all the four fruitflies as function of flight speed. From the overall data in Figure 13a, a clear trend of $\Delta \Phi$ can be observed that $\Delta \Phi$ thus $\Phi$ first decreases ( $\Phi$ is about $8 \%$ smaller at $V_{\infty} \approx 0.7 \mathrm{~m} / \mathrm{s}$ than that at near-hovering case) and then increases ( $\Phi$ is about $16 \%$ larger at the maximum flight speed than that at near-hovering case) with flight speed increasing. From the overall data in Figure 13b, it can be seen that 
$\bar{\phi}$ increases with flight speed increasing in the speed range of $V_{\infty} \approx 0-0.7 \mathrm{~m} / \mathrm{s}$, but remains unchanged and even decreases a little at higher flight speeds. In addition, $d / u$ (Table 2 ) is approximately 1.1 at near-hovering and gradually increases with flight speed increasing. At the highest flight speeds of FF1 and FF2, $d / u$ are close to 1.6 , resulting in a relatively large flapping velocity of the wing relative to the body in upstroke in comparison to that in downstroke.
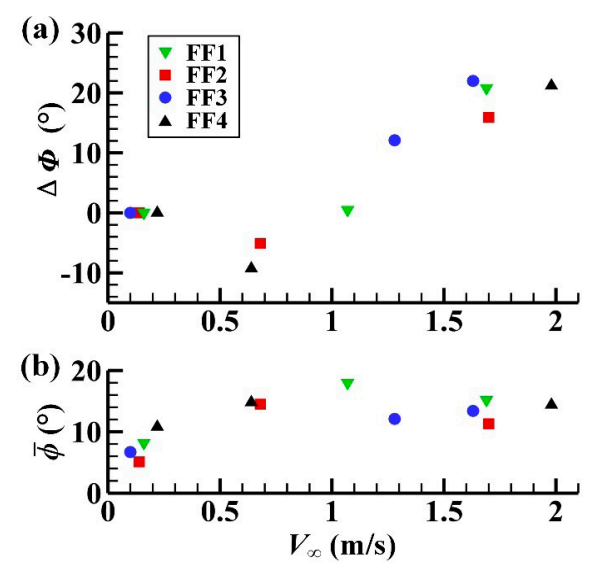

Figure 13. The increments of (a) the stroke amplitude and (b) mean stroke angle as function of flight speed for all the four fruitflies.

\subsection{Aerodynamic Forces}

\subsubsection{Code Validation and Grid Test}

The flow solver had been validated in our previous studies by comparing the computed unsteady aerodynamic forces with the measured results of model insect wings in translating [42], revolving and flapping motions [43]. These tests all showed that the computed aerodynamic forces by the present solver were in good agreement with the corresponding experimental measurements.

To further ensure the computation is grid-independent, a grid resolution test was conducted using the wing models and wing kinematics of FF1 flying at medium speed $\left(V_{\infty}=1.07 \mathrm{~m} / \mathrm{s}\right)$. Three grid systems were considered. For the grid system 1 , the dimensions of the wing grid were $31 \times 45 \times 37$ in the normal direction, around the wing and in the spanwise direction, respectively (the first-layer thickness was 0.002c); the dimensions of the background grid were $101 \times 61 \times 61$ in the $x, y$ and $z$ directions, respectively. For grid system 2, the corresponding grid dimensions were $45 \times 67 \times 57$ $(0.0015 \mathrm{c})$ and $155 \times 95 \times 95$. For grid system 3 , the corresponding grid dimensions were $66 \times 101 \times 83$ (0.001c) and $234 \times 141 \times 141$. For the wing grids in these grid systems, the grid points were densely distributed toward the wing surface and toward the wake area; for the background grids, the grid points were concentrated in the near field of the wings. The non-dimensional time step was 0.02 .

Shown in Figure 14a,b are the vertical force and thrust coefficients in a wingbeat cycle, computed with the above three grid systems. Plotted in Figure $14 \mathrm{c}$ are the iso-vorticity surfaces of the non-dimensional vorticity at $\tau=0.42$. It can be found that there is almost no difference between the aerodynamic force coefficients calculated by the three grid systems; and some discrepancies exist in the iso-surface plots of vorticity between grid system 2 and grid system 1, but there are only very small discrepancies between grid system 2 and grid system 3 . This indicates that good solution accuracy can be achieved with grid system 2 or 3 . Here, grid system 3 was used in the present paper. 

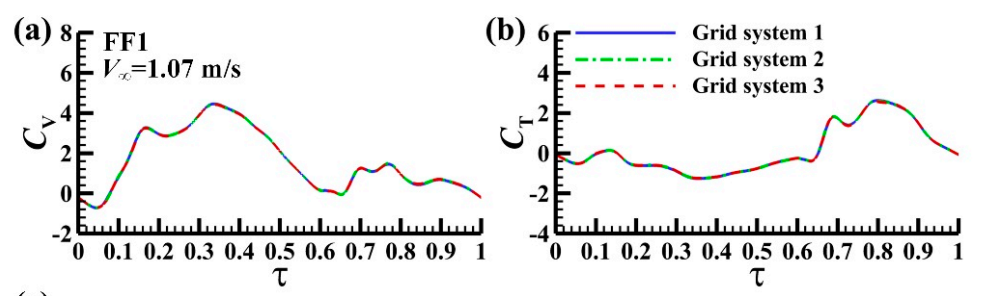

(c)

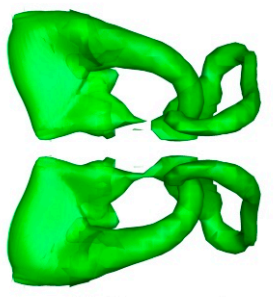

Grid system 1

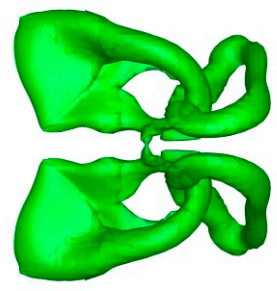

Grid system 2

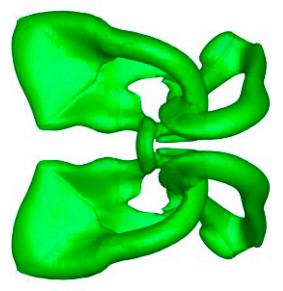

Grid system 3

Figure 14. Grid resolution test. Time courses of the (a) vertical force and (b) thrust coefficients of the wings in a wingbeat cycle computed with three grid systems. (c) The iso-surface plots of the non-dimensional vorticity at $\tau=0.42$ (the magnitude of the non-dimensional vorticity is 1 ).

\subsubsection{Computed Aerodynamic forces}

Using the numerical method described above and the measured wing kinematics, the aerodynamic forces at each flight of the fruitflies can be computed. Figure 15 shows the vertical force $\left(C_{V}\right)$ and thrust $\left(C_{\mathrm{T}}\right)$ coefficients on the wings in a wingbeat cycle of $\mathrm{FF} 1$ at three flight speeds, after periodical state has been achieved (the corresponding results of the other three fruitflies are presented in Figure A5 in the Appendix A).
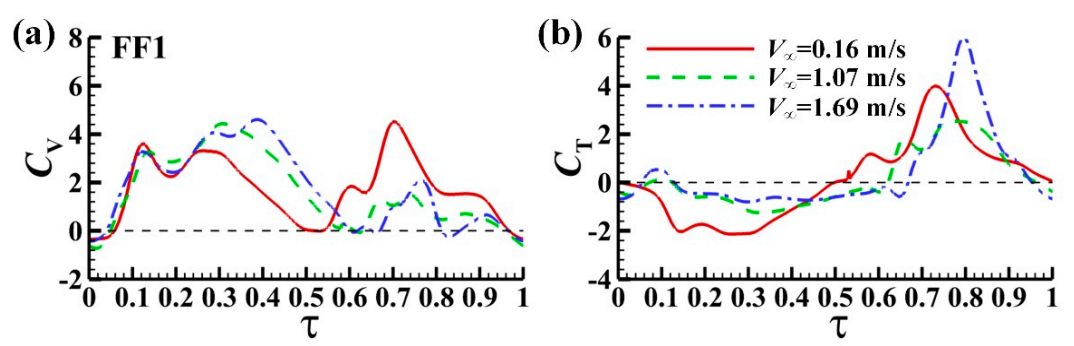

Figure 15. Time courses of the computed (a) vertical force and (b) thrust coefficients in a wingbeat cycle of FF1 at three flight speeds.

Figure 16 shows the instantaneous force vectors at several moments in one cycle superimposed on the wing-tip paths for FF1 at three flight speeds. The following observations can be made from Figures 15 and 16.

At near-hovering $\left(V_{\infty}=0.16 \mathrm{~m} / \mathrm{s}\right)$, positive $C_{\mathrm{V}}$ is produced in downstroke and upstroke to support the weight (Figures 15a and 16a), and the contribution of downstroke is about $53 \%$, only slightly larger than that of upstroke. Negative $C_{\mathrm{T}}$ produced in downstroke and positive in upstroke (Figures 15b and 16a) have almost the same magnitude that cancel each other out, resulting in an approximately zero mean thrust, as required for the hovering flight. At medium flight speed $\left(V_{\infty}=1.07 \mathrm{~m} / \mathrm{s}\right)$, positive $C_{\mathrm{V}}$ produced in downstroke is about $86 \%$ of the total $C_{\mathrm{V}}$ in the entire cycle, much larger than its counterpart in upstroke (Figure 15a). Thus, the weight-supporting force is mainly generated during downstroke (Figure 16b). The magnitudes of positive $C_{\mathrm{T}}$ produced in upstroke and negative $C_{\mathrm{T}}$ produced in downstroke are both decreased, but the magnitude of the positive $C_{\mathrm{T}}$ in upstroke is much larger than its negative counterpart in downstroke (Figure 15b), resulting in a positive cycle-averaged thrust. Thus, the thrust that required for overcoming the body drag is mainly generated in upstroke (Figure 16b). 


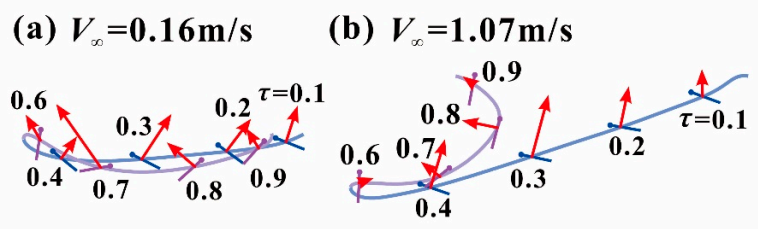

(c) $V_{s}=1.69 \mathrm{~m} / \mathrm{s}$

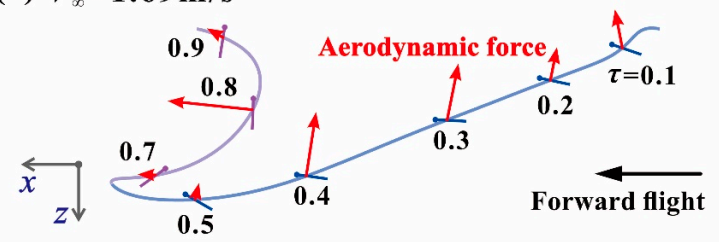

Figure 16. Instantaneous force vectors (red arrows) superimposed on the side view of the wing-tip paths for FF1 at three flight speeds. The blue and purple lines indicate the orientation of the wing in downstroke and upstroke, respectively, at several times in a flapping cycle, with dots marking the leading edge. (a) At near-hovering $\left(V_{\infty}=0.16 \mathrm{~m} / \mathrm{s}\right)$; (b) At medium flight speed $\left(V_{\infty}=1.07 \mathrm{~m} / \mathrm{s}\right)$; (c) At high flight speed $\left(V_{\infty}=1.69 \mathrm{~m} / \mathrm{s}\right)$.

At high flight speed $\left(V_{\infty}=1.69 \mathrm{~m} / \mathrm{s}\right)$, positive $C_{\mathrm{V}}$ produced in downstroke is rather larger and in upstroke is rather smaller (Figures 15a and 16c). About $89 \%$ of the weight-supporting vertical force is produced during downstroke. Same as the medium-speed case, the thrust required for overcoming the larger body drag is mainly generated in upstroke (Figures 15b and 16c). The similar observations of FF2-FF4 are given in Appendix A.

For steady forward flight, the aerodynamic forces acting on the wings and the body should be approximately equal to the insect-weight in the vertical direction and should be close to zero in the horizontal direction. The non-dimensional weight $\left(C_{\mathrm{G}}=m g / 0.5 \rho U^{2}(2 S)\right.$, in which $g$ is the acceleration of gravity) of FF1 is about 1.76. The computed non-dimensional mean vertical force of the wings at three flight speeds for FF1 are 1.80, 1.75 and 1.62, respectively. At $V_{\infty}=0.16$ and $1.07 \mathrm{~m} / \mathrm{s}$, the differences of the computed vertical forces on the wings, compared with $C_{\mathrm{G}}$, are less than $2 \%$, which means the computed vertical forces on the wings are sufficient for supporting the weight. At $V_{\infty}=1.69 \mathrm{~m} / \mathrm{s}$, as the body angle is about $27^{\circ}$, the small vertical force on the body would compensate the shortage, about $8 \%$, between the computed vertical force acting on the wings and $C_{\mathrm{G}}$. Therefore, the equilibrium conditions that the vertical forces balance the weight at all three flight speeds of FF1 are approximately satisfied. The similar results of FF2-FF4 are presented in the Appendix A.

\subsection{Power Requirements}

\subsubsection{Computed Mechanical Power}

Power requirement is a crucial parameter to quantify the flight performance of insect. Based upon the measured wing kinematics and the computed aerodynamic forces, the aerodynamic and inertial powers can be calculated using the computational method described in Section 2.4. Figure 17 shows the time histories of the instantaneous non-dimensional aerodynamic $\left(C_{\mathrm{Pa}}\right)$, inertial $\left(C_{\mathrm{Pi}}\right)$ and mechanical $\left(C_{P}\right)$ powers in one stroke cycle of FF1-FF4 at various flight speeds. It can be seen that at all flight speeds of these fruitflies, $C_{\mathrm{Pi}}$ is much smaller than $C_{\mathrm{Pa}}$ in most of the flapping cycle, and hence the curves of $C_{\mathrm{P}}$ are approximately the same as that of $C_{\mathrm{Pa}}$. Moreover, the value of $C_{\mathrm{Pi}}$ is very small (close to zero) in downstroke and is positive in the first half of upstroke and is negative in the next half of upstroke. This indicates that for fruitflies in near-hovering or forward flights, the influence of inertial power on power consumption (the cycle-averaged mechanical power) is very small. 

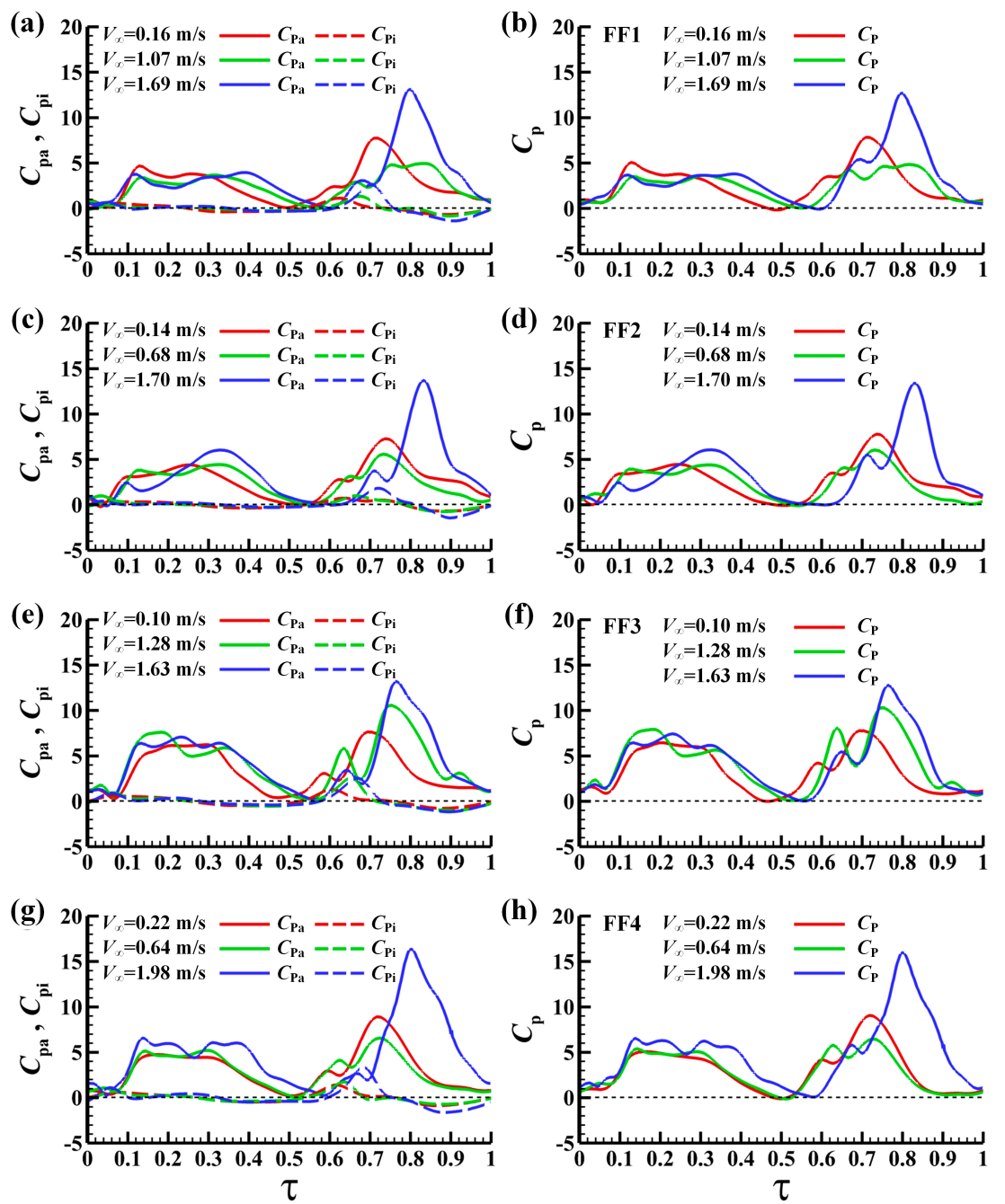

Figure 17. Time histories of $(\mathbf{a}, \mathbf{c}, \mathbf{e}, \mathbf{g}) C_{\mathrm{Pa}}$ and $C_{\mathrm{Pi}}$ and $(\mathbf{b}, \mathbf{d}, \mathbf{f}, \mathbf{h}) C_{\mathrm{P}}$ in one stroke cycle of FF1-FF4 at various flight speeds.

As seen from Figure 17, the magnitude of $C_{P}$ changes slightly in downstroke and significantly in upstroke for all the fruitflies with flight speed increasing. Compared with their respective near-hovering cases, the peak values of $C_{\mathrm{P}}$ in upstroke at low or medium flight speeds (e.g., FF1 at $V_{\infty}=1.07 \mathrm{~m} / \mathrm{s}$, FF2 at $V_{\infty}=0.68 \mathrm{~m} / \mathrm{s}$ and FF4 at $V_{\infty}=0.64 \mathrm{~m} / \mathrm{s}$ ) are a little smaller but at higher flight speeds are much larger. Thus, it can be concluded that the magnitude of $C_{P}$ in upstroke first decreases and then increases with flight speed increasing.

In order to analyze how $C_{\mathrm{Pa}}$ (or $C_{\mathrm{P}}$ ) is generated, here we take the flights of FF1 as examples and the interpretation is as follows. As seen from Equation (5), $C_{\mathrm{Pa}}$ is the inner product of $\boldsymbol{M}_{\mathrm{a}}$ and $\Omega$. Since the wings of FF1 are approximately flapping back and forth in the stroke plane at all three flight speeds, the aerodynamic power consumed by a wing is mainly used to overcome the drag exerted on it (the wing drag is the force that the insect must overcome for moving its wing). Therefore, the aerodynamic power must be positive (Figure 17) and is closely related to $C_{\mathrm{D}}$ and $\dot{\phi}$ (where "dot" denotes a time derivative). Figure 18 shows the time histories of $C_{\mathrm{D}}$ and $\dot{\phi}$ in a wingbeat cycle of FF1 at three flight speeds. As illustrated in Figures 17 and 18, in the first half downstroke, $C_{\mathrm{Pa}}$ at near-hovering $\left(V_{\infty}=0.16 \mathrm{~m} / \mathrm{s}\right)$ is a little larger than that at medium $\left(V_{\infty}=1.07 \mathrm{~m} / \mathrm{s}\right)$ and high $\left(V_{\infty}=1.69 \mathrm{~m} / \mathrm{s}\right)$ flight speeds due to the larger magnitude of $\dot{\phi}$; while in the next half downstroke, $C_{\mathrm{Pa}}$ increases with flight speed due to the increasing of the magnitudes of $C_{\mathrm{D}}$ and $\dot{\phi}$. During upstroke, from near-hovering to medium flight speed, the magnitude of $C_{\mathrm{D}}$ decreases a little, thus $C_{\mathrm{Pa}}$ decreases a little; whereas at 
high flight speeds, both of the magnitudes of $C_{\mathrm{D}}$ and $\dot{\phi}$ increase significantly, thus $C_{\mathrm{Pa}}$ becomes very large. Additionally, because of $d / u$ is increasing with flight speed, the peak position of $C_{P}$ in upstroke, where the values of $C_{\mathrm{D}}$ and $\dot{\phi}$ are maximal, continues to be delayed. Through the same method of analysis, the similar results of FF2-FF4 can also be obtained.
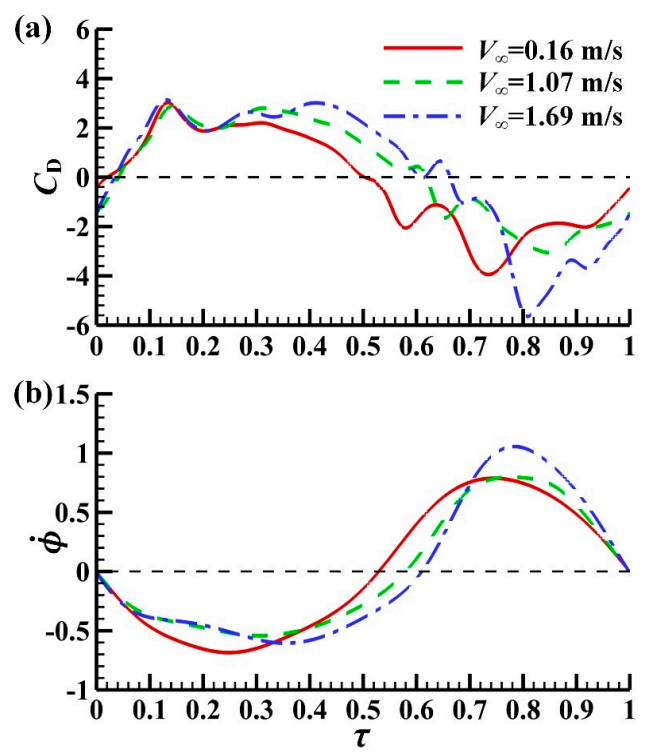

Figure 18. Time histories of drag coefficient and flapping angular velocity of the wing in a wingbeat cycle of FF1 at three flight speeds. (a) Drag coefficient; (b) Flapping angular velocity.

\subsubsection{Specific power}

To evaluate the power requirement of insect flight, the body-mass-specific power $\left(P^{*}\right.$, defined as the cycle-averaged mechanical power divided by the mass of the insect) is usually used:

$$
P^{*}=0.5 \rho U^{3}(2 S) \times\left(C_{W} / T^{*}\right) / m
$$

where $C_{W}$ is the non-dimensional work in a wingbeat cycle and $T^{*}$ is the non-dimensional period (defined as $T^{*}=U / c f$ ).

Commonly, when computing $C_{W}$, two limiting cases are considered [44]: in the case of zero elastic energy storage, with the added assumption that the cost for dissipating the negative work is negligible, $C_{W}$ is calculated by integrating $C_{P}$ over the part of a wingbeat cycle where it is positive; in the case of $100 \%$ elastic energy storage, with the added assumption that the negative work can be stored by an elastic element and then can be released when the wing does positive work, $C_{W}$ is calculated by integrating $C_{\mathrm{P}}$ over the entire wingbeat cycle. The real $C_{\mathrm{W}}$ lies between the values of these two limiting cases. However, for all the flights of the four fruitflies in the present paper, $C_{P}$ is positive in almost the entire flapping cycle because the value of negative $C_{\mathrm{Pi}}$ is very small. Consequently, $C_{\mathrm{W}}$ calculated by these two considered limiting cases will be approximately the same and any of them is a good approximation of the real $C_{W}$. This result is similar to that in previous studies $[45,46]$. Here, the case of $100 \%$ elastic energy storage is adopted.

With $C_{\mathrm{W}}$ known, $P^{*}$ at each flight of FF1-FF4 can be calculated using Equation (6), and the results as function of advance ratio $(J)$ are plotted in Figure 19. The specific powers at near-hovering of FF1-FF4 (the first data point of each fruitfly) are about $26.6 \mathrm{~W} / \mathrm{kg}$. And the variations of $P^{*}$ with flight speed of the four fruitflies are consistent. From the overall data of the specific powers, it can be observed that $P^{*}$ only changes very little when the advance ratio changes from 0 to about 0.45 , and afterwards $P^{*}$ increases sharply. 


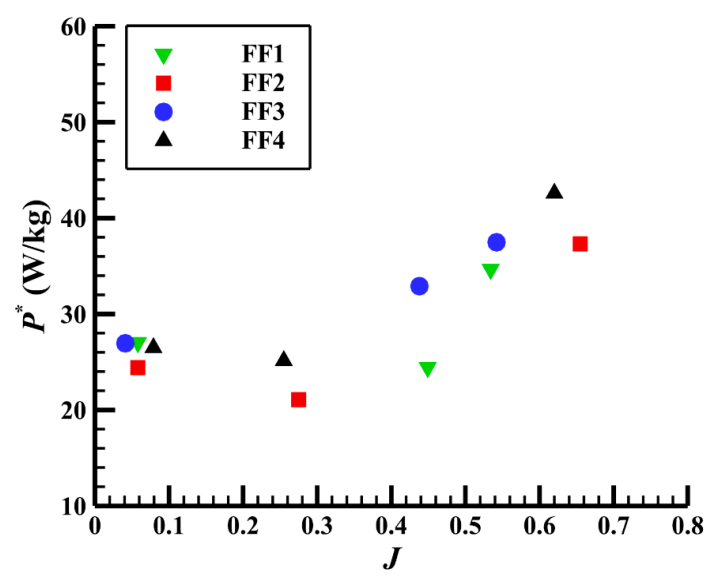

Figure 19. Specific power versus advance ratio of fruitflies.

For example, the specific power of FF4 at the highest flight speed is approximately $61 \%$ larger than that at near-hovering. That is, the energy consumption of the fruitflies varies with flight speed according to a J-shaped curve. This is similar to that of the medium-sized bumblebee [23] but different to that of the large-sized hawkmoth which is U-shaped [7]. One possible reason for this difference may be that the size of the hawkmoth is much larger and flapping frequency much lower than those of the fruitfly and bumblebee (hawkmoth: wing-length about $50 \mathrm{~mm}$ and frequency about $25 \mathrm{~Hz}$; fruitfly and bumblebee: wing-length 3-13 $\mathrm{mm}$ and frequency $150-200 \mathrm{~Hz}$ ).

\section{Conclusions}

In this paper, an elaborate filming system consisting of three high-speed cameras, a customized low-speed open-jet wind tunnel and other visual controlling devices was designed to capture the forward flight of fruitflies. The wing and body kinematics at multiple speeds of four fruitflies flying in their full speed range were successfully measured, and the maximum flight speed obtained was about $2 \mathrm{~m} / \mathrm{s}$, reaching the limiting flight speed of fruitfly. The kinematic results show that, as flight speed increases, the body angle decreases and the stroke plane angle increases; the fluctuation of the wingbeat frequency is small; the geometrical angle of attack in the middle upstroke increases while in the middle downstroke almost remains unchanged; the stroke amplitude decreases first and then increases; the mean stroke angle increases in the speed range of $V_{\infty} \approx 0-0.7 \mathrm{~m} / \mathrm{s}$ but remains unchanged and even decreases a little at higher flight speeds; the ratio of downstroke duration to upstroke duration increases. The aerodynamic forces and power requirement at each flight speed were computed using CFD method. It is shown that the mechanical power is dominated by aerodynamic power, and the magnitude of aerodynamic power in upstroke increases significantly at high flight speeds due to the increase of the drag and the flapping velocity of the wing. The overall data of the specific powers of the four fruitflies as function of advance ratio shows that the specific power only changes a little when the advance ratio is below about 0.45 and afterwards increases sharply. That is, the energy consumption of the fruitflies varies with flight speed according to a J-shaped curve.

Supplementary Materials: The following are available online at http://www.mdpi.com/1996-1073/13/16/4271/s1, Video S1: FF1 near hovering, Video S2: FF1 medium speed, Video S3: FF1 high speed.

Author Contributions: Conceptualization, H.J.Z. and M.S.; Methodology, H.J.Z. and M.S.; Software, H.J.Z.; Validation, H.J.Z.; Formal Analysis, H.J.Z. and M.S.; Investigation, H.J.Z.; Resources, H.J.Z.; Data Curation, H.J.Z.; Writing-Original Draft Preparation, H.J.Z.; Writing-Review \& Editing, M.S.; Visualization, H.J.Z.; Supervision, M.S.; Project Administration, H.J.Z.; Funding Acquisition, M.S. All authors have read and agreed to the published version of the manuscript.

Funding: This research was funded by the National Natural Science Foundation of China, grant numbers 11232030 and 11832004.

Conflicts of Interest: The authors declare no conflict of interest. 


\section{Appendix A}

The measured time courses of stroke angle, deviation angle and pitch angle in a wingbeat cycle and the corresponding wing-tip path of FF2-FF4 at all flight speeds are given in Figures A1-A3, respectively.
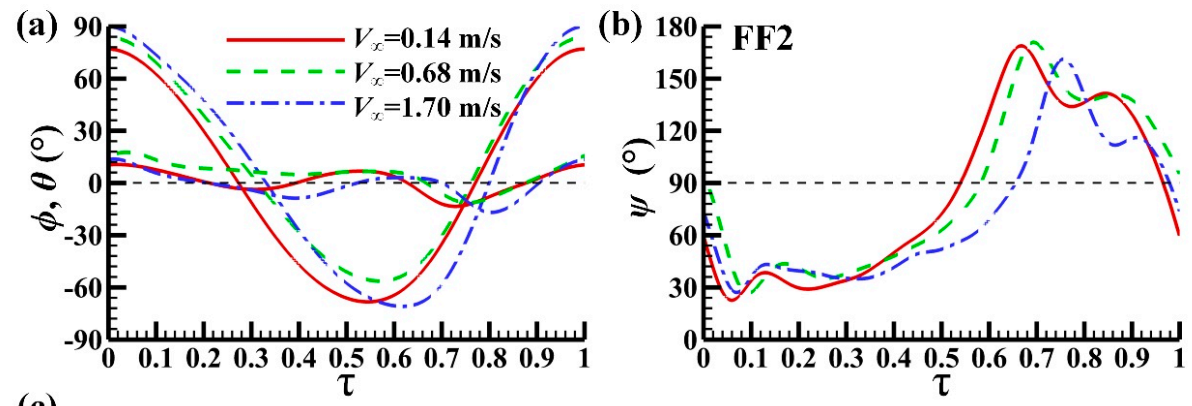

(c)

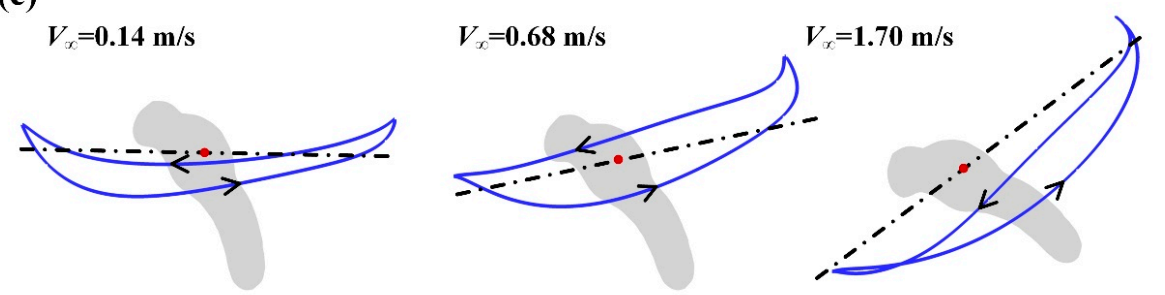

Figure A1. Time courses of (a) $\phi$ and $\theta$ and (b) $\psi$ for FF2 in one wingbeat cycle at three flight speeds. (c) The wing-tip paths relative to the stroke plane determined by $\phi$ and $\theta$ at each flight speed (the stroke plane is indicated by the dash-dot line and the wing-root is indicated by a red dot).
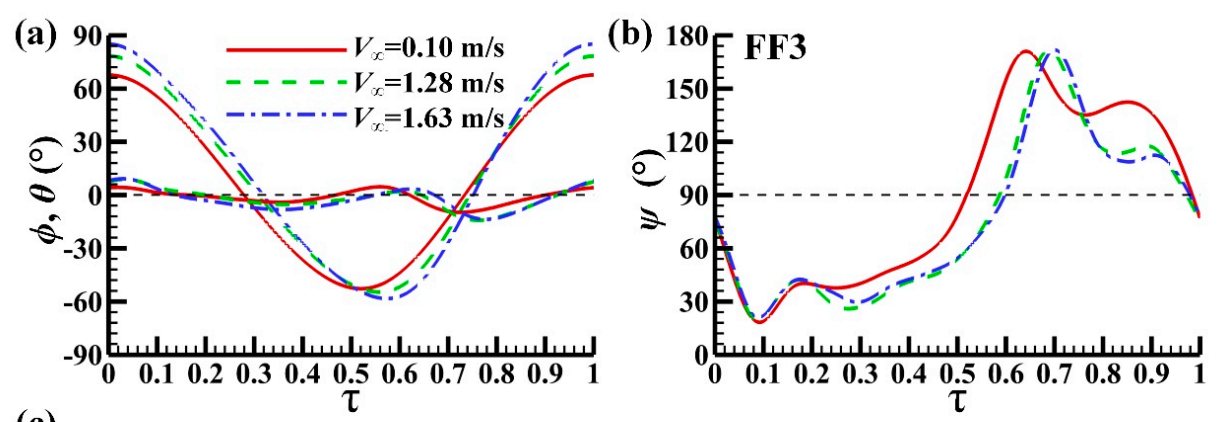

(c)
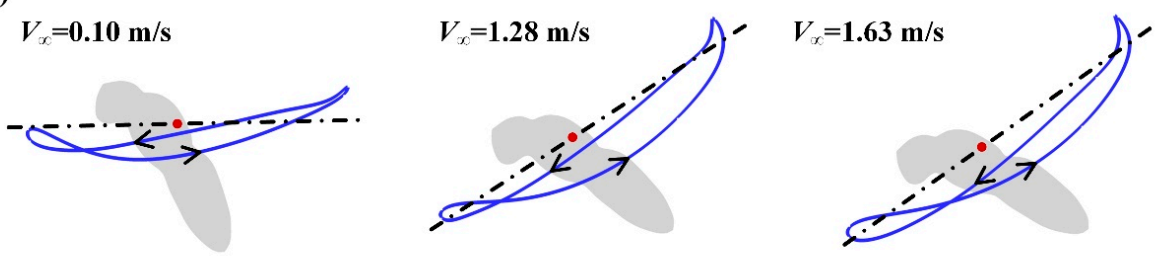

Figure A2. Time courses of (a) $\phi$ and $\theta$ and (b) $\psi$ for FF3 in one wingbeat cycle at three flight speeds. (c) The wing-tip paths relative to the stroke plane determined by $\phi$ and $\theta$ at each flight speed (the stroke plane is indicated by the dash-dot line and the wing-root is indicated by a red dot).

Time courses of geometrical angle of attack in a wingbeat cycle of FF2-FF4 at all flight speeds are given in Figure A4. As flight speed increases, it can be observed that the variations of $\alpha$ of FF2-FF4 are similar to that of FF1 (described in Section 3.1). 

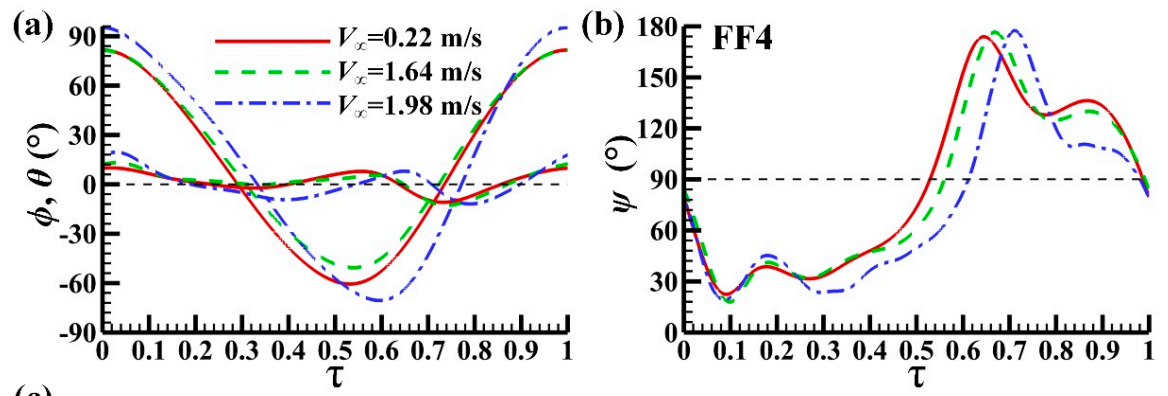

(c)

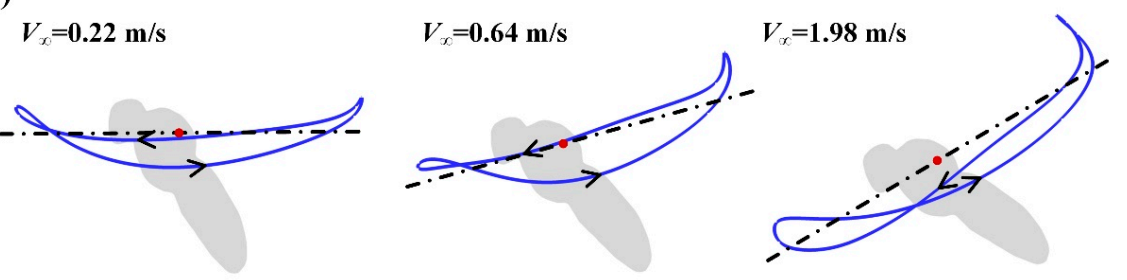

Figure A3. Time courses of (a) $\phi$ and $\theta$ and (b) $\psi$ for FF4 in one wingbeat cycle at three flight speeds. (c) The wing-tip paths relative to the stroke plane determined by $\phi$ and $\theta$ at each flight speed (the stroke plane is indicated by the dash-dot line and the wing-root is indicated by a red dot).
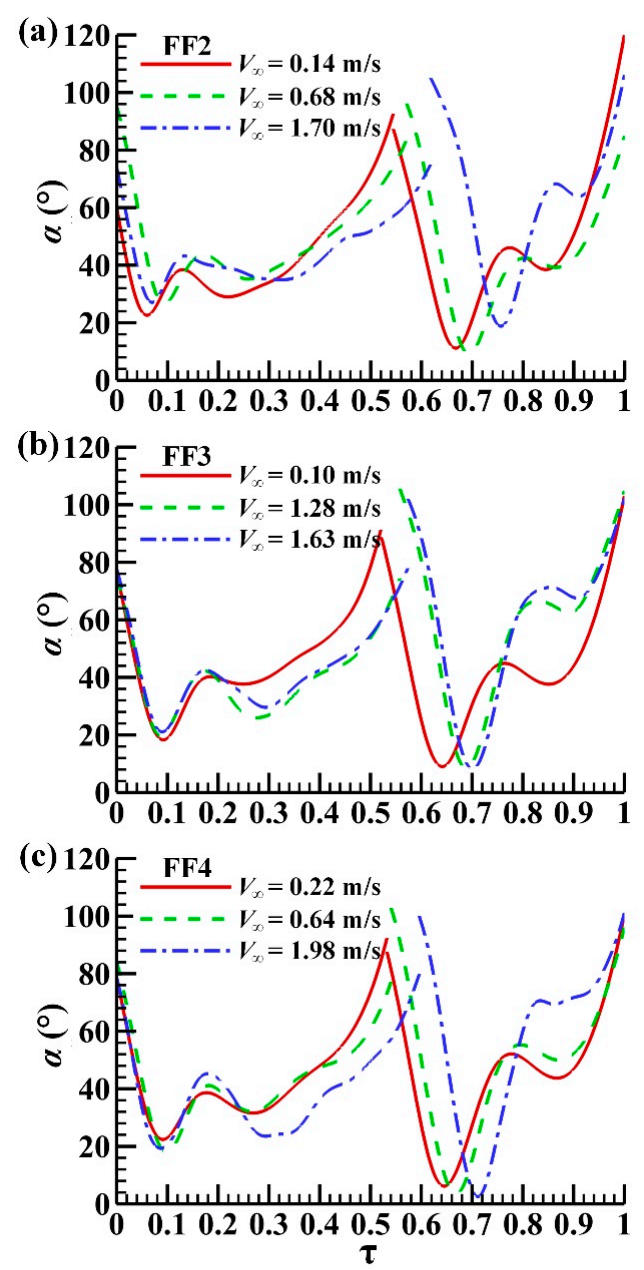

Figure A4. Time courses of $\alpha$ in a wingbeat cycle for FF2-FF4 at various flight speeds. (a) FF2; (b) FF3; (c) FF4.

The computed $C_{\mathrm{V}}$ and $C_{\mathrm{T}}$ on the wings in a flapping cycle after periodical state has been established for FF2-FF4 at various flight speeds are shown in Figure A5. The following observations which is 
similar to that of FF1 can be made. At near hovering, both of downstroke and upstroke contribute to the weight-supporting vertical force, and the contributions are approximately the same; positive $C_{\mathrm{T}}$ in upstroke and negative in downstroke cancel each other out, bringing an equilibrium state. As flight speed increases, the contribution of downstroke to $C_{V}$ to support the weight is increasing, while the contribution of upstroke to $C_{\mathrm{T}}$ to overcome the body drag is increasing.
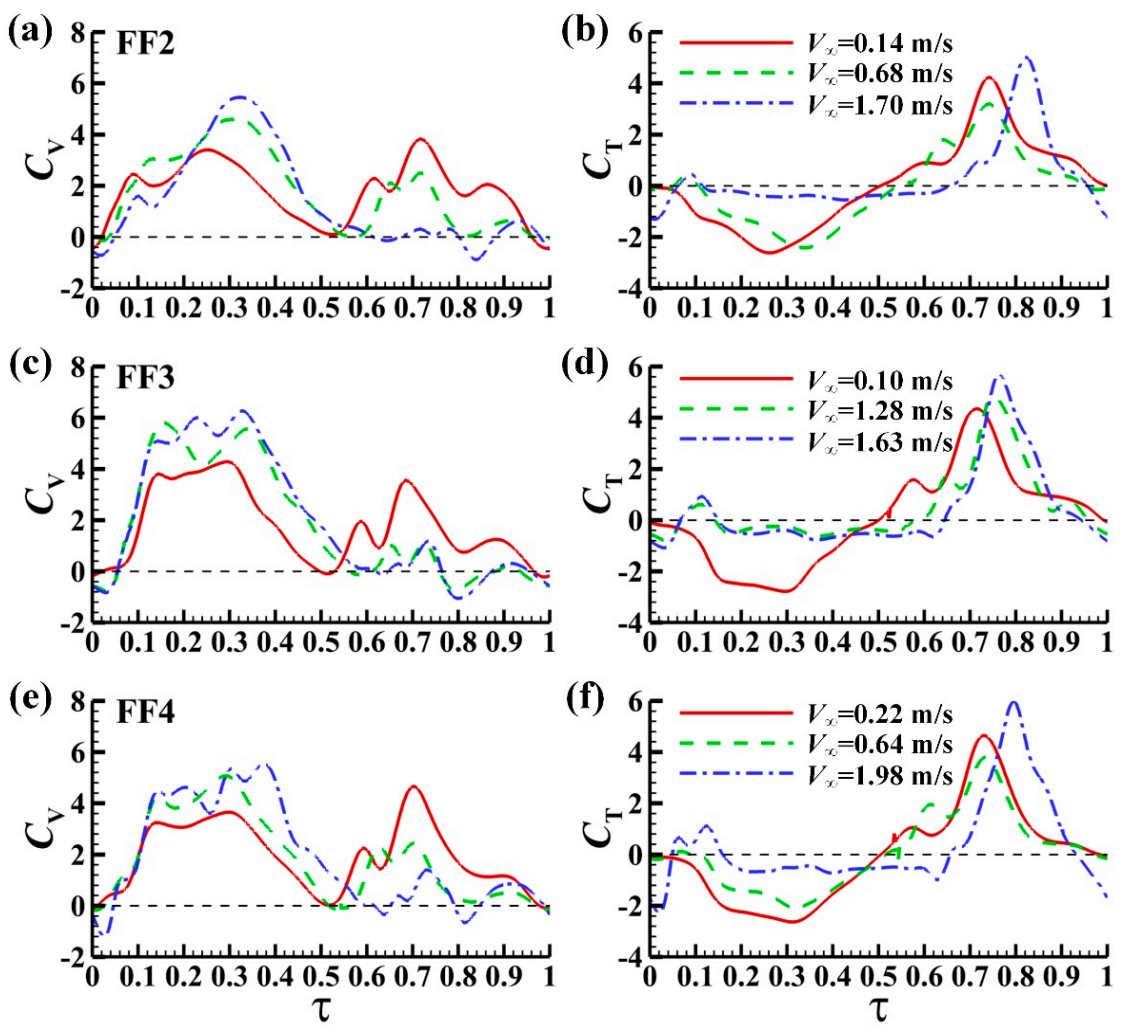

Figure A5. Time courses of the computed $(\mathbf{a}, \mathbf{c}, \mathbf{e})$ vertical force and $(\mathbf{b}, \mathbf{d}, \mathbf{f})$ thrust coefficients in a wingbeat cycle of FF2-FF4 at various flight speeds.

\section{References}

1. Dudley, R. The Biomechanics of Insect Flight: Form, Function, Evolution; Princeton University Press: Princeton, NJ, USA, 2002; p. 496.

2. Weis-Fogh, T. Energetics of hovering flight in hummingbirds and in Drosophila. J. Exp. Biol. 1972, 56, 79-104.

3. Pennycuick, C.J. Power requirements for horizontal flight in the pigeon Columba Livia. J. Exp. Biol. 1968, 49, 527-555.

4. Pennycuick, C.J. Mechanics of flight. In Avian Biology; Farner, D.S., King, J.R., Parkes, K.C., Eds.; Academic Press: New York, NY, USA, 1975; Volume 5, pp. 1-75.

5. Dickinson, M.H.; Lehmann, F.-O.; Chan, W.P. The control of mechanical power in insect flight. Am. Zool. 1998, 38, 718-728. [CrossRef]

6. Ellington, C.P.; Machin, K.E.; Casey, T.M. Oxygen consumption of bumblebees in forward flight. Nature 1990, 347, 472-473. [CrossRef]

7. Warfvinge, K.; KleinHeerenbrink, M.; Hedenstrom, A. The power-speed relationship is U-shaped in two free-flying hawkmoths (Manduca sexta). J. R. Soc. Interface 2017, 14, 20170372. [CrossRef]

8. Ellington, C.P. The aerodynamics of hovering insect flight. III. Kinematics. Philos. Trans. R. Soc. Lond. Ser. B Biol. Sci. 1984, 305, 41-78. [CrossRef]

9. Fry, S.N.; Sayaman, R.; Dickinson, M.H. The aerodynamics of hovering flight in Drosophila. J. Exp. Biol. 2005, 208, 2303-2318. [CrossRef]

10. Ristroph, L.; Berman, G.J.; Bergou, A.J.; Wang, Z.J.; Cohen, I. Automated hull reconstruction motion tracking (HRMT) applied to sideways maneuvers of free-flying insects. J. Exp. Biol. 2009, 212, 1324-1335. [CrossRef] 
11. Liu, Y.P.; Sun, M. Wing kinematics measurement and aerodynamics of hovering droneflies. J. Exp. Biol. 2008, 211, 2014-2025. [CrossRef]

12. Cheng, B.; Deng, X.Y.; Hedrick, T.L. The mechanics and control of pitching manoeuvres in a freely flying hawkmoth (Manduca sexta). J. Exp. Biol. 2011, 214, 4092-4106. [CrossRef]

13. Meng, X.G.; Sun, M. Aerodynamics and vortical structures in hovering fruitflies. Phys. Fluids 2015, $27,031901$. [CrossRef]

14. Meng, X.G.; Sun, M. Wing kinematics, aerodynamic forces and vortex-wake structures in fruit-flies in forward flight. J. Bionic Eng. 2016, 13, 478-490. [CrossRef]

15. Zou, P.Y.; Lai, Y.H.; Yang, J.T. Effects of phase lag on the hovering flight of damselfly and dragonfly. Phys. Rev. E 2019, 100, 063102. [CrossRef] [PubMed]

16. Lyu, Y.Z.; Zhu, H.J.; Sun, M. Flapping-mode changes and aerodynamic mechanisms in miniature insects. Phys. Rev. E 2019, 99, 012419. [CrossRef] [PubMed]

17. Lyu, Y.Z.; Zhu, H.J.; Sun, M. Wing kinematic and aerodynamic compensations for unilateral wing damage in a small phorid fly. Phys. Rev. E 2020, 101, 012412. [CrossRef]

18. Liu, L.G.; Du, G.; Sun, M. Aerodynamic-force production mechanisms in hovering mosquitoes. J. Fluid Mech. 2020, 898, A19. [CrossRef]

19. Li, X.; Guo, C. Wing-kinematics measurement and flight modelling of the bamboo weevil C. buqueti. IET Nanobiotechnol. 2020, 14, 53-58. [CrossRef]

20. Dudley, R.; Ellington, C.P. Mechanics of forward flight in bumblebees: I. Kinematics and morphology. J. Exp. Biol. 1990, 148, 19-52.

21. Willmott, A.P.; Ellington, C.P. The mechanics of flight in the hawkmoth Manduca sexta. I. Kinematics of hovering and forward flight. J. Exp. Biol. 1997, 200, 2705-2722.

22. Meng, X.G.; Sun, M. Wing and body kinematics of forward flight in drone-flies. Bioinspir. Biomim. 2016, 11, 056002. [CrossRef]

23. Wu, J.H.; Sun, M. Unsteady aerodynamic forces and power requirements of a bumblebee in forward flight. Acta Mech. Sin. 2005, 21, 207-217. [CrossRef]

24. David, C.T. Compensation for height in the control of groundspeed by Drosophila in a new, 'barber's pole' wind tunnel. J. Comp. Physiol. A 1982, 147, 485-493. [CrossRef]

25. Fry, S.N.; Rohrseitz, N.; Straw, A.D.; Dickinson, M.H. Visual control of flight speed in Drosophila melanogaster. J. Exp. Biol. 2009, 212, 1120-1130. [CrossRef] [PubMed]

26. Fuller, S.B.; Straw, A.D.; Peek, M.Y.; Murray, R.M.; Dickinson, M.H. Flying Drosophila stabilize their vision-based velocity controller by sensing wind with their antennae. Proc. Natl. Acad. Sci. USA 2014, 111, 1182-1191. [CrossRef] [PubMed]

27. Fry, S.N.; Sayaman, R.; Dickinson, M.H. The aerodynamics of free-flight maneuvers in Drosophila. Science 2003, 300, 495-498. [CrossRef]

28. Vogel, S. Flight in Drosophila: I. Flight performance of tethered flies. J. Exp. Biol. 1966, 44, 567-578.

29. Bartussek, J.; Lehmann, F.O. Proprioceptive feedback determines visuomotor gain in Drosophila. R. Soc. Open Sci. 2016, 3, 150562. [CrossRef]

30. Mou, X.L.; Liu, Y.P.; Sun, M. Wing motion measurement and aerodynamics of hovering true hoverflies. J. Exp. Biol. 2011, 214, 2832-2844. [CrossRef]

31. Aono, H.; Liang, F.; Liu, H. Near- and far-field aerodynamics in insect hovering flight: An integrated computational study. J. Exp. Biol. 2008, 211, 239-257. [CrossRef]

32. Yu, X.; Sun, M. A computational study of the wing-wing and wing-body interactions of a model insect. Acta Mech. Sin. 2009, 25, 421-431. [CrossRef]

33. Liang, B.; Sun, M. Aerodynamic interactions between wing and body of a model insect in forward flight and maneuvers. J. Bionic Eng. 2013, 10, 19-27. [CrossRef]

34. Rogers, S.E.; Kwak, D. Upwind differencing scheme for the time-accurate incompressible Navier-Stokes equations. AIAA J. 1990, 28, 253-262. [CrossRef]

35. Rogers, S.; Kwak, D. Numerical solution of the incompressible Navier-Stokes equations for steady-state and time-dependent problems. In Proceedings of the 27th Aerospace Sciences Meeting, Reno, NV, USA, 9-12 January 1989. 
36. Hilgenstock, A. A fast method for the elliptic generation of three-dimensional grids with full boundary control. In Proceedings of the 2nd International Conference on Numerical Grid Generation in Computational Fluid Mechanics, Miami, FL, USA, 5-9 December 1988; pp. 137-146.

37. Sun, M. Insect flight dynamics: Stability and control. Rev. Mod. Phys. 2014, 86, 615-646. [CrossRef]

38. Xiong, Y.; Sun, M. Dynamic flight stability of a bumblebee in forward flight. Acta Mech. Sin. 2008, 24, 25-36. [CrossRef]

39. Xu, N.; Sun, M. Lateral dynamic flight stability of a model bumblebee in hovering and forward flight. J. Theor. Biol. 2013, 319, 102-115. [CrossRef]

40. Zhu, H.J.; Meng, X.G.; Sun, M. Forward flight stability in a drone-fly. Sci. Rep. 2020, 10, 1975. [CrossRef]

41. Yao, J.; Yeo, K.S. Forward flight and sideslip manoeuvre of a model hawkmoth. J. Fluid Mech. 2020, 896, A22. [CrossRef]

42. Meng, X.G.; Sun, M. Aerodynamic effects of wing corrugation at gliding flight at low Reynolds numbers. Phys. Fluids 2013, 25, 071905. [CrossRef]

43. Cheng, X.; Sun, M. Very small insects use novel wing flapping and drag principle to generate the weight-supporting vertical force. J. Fluid Mech. 2018, 855, 646-670. [CrossRef]

44. Ellington, C.P. The aerodynamics of hovering insect flight. VI. Lift and power requirements. Philos. Trans. R. Soc. Lond. Ser. B Biol. Sci. 1984, 305, 145-181.

45. Sun, M.; Tang, J. Lift and power requirements of hovering flight in Drosophila virilis. J. Exp. Biol. 2002, 205, 2413-2427. [PubMed]

46. Sun, M.; Du, G. Lift and power requirements of hovering insect flight. Acta Mech. Sin. 2003, 19, 458-469.

(C) 2020 by the authors. Licensee MDPI, Basel, Switzerland. This article is an open access article distributed under the terms and conditions of the Creative Commons Attribution (CC BY) license (http://creativecommons.org/licenses/by/4.0/). 